\title{
A Digital Twin for Friction Prediction in Dynamic Rubber Applications with Surface Textures
}

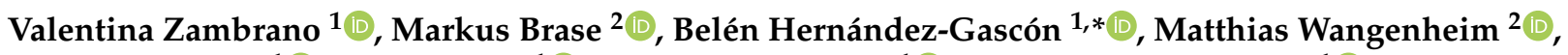 \\ Leticia A. Gracia ${ }^{1}{ }^{\mathbb{D}}$, Ismael Viejo ${ }^{1}{ }^{\mathbb{D}}$, Salvador Izquierdo ${ }^{1} \mathbb{D}$ and José Ramón Valdés ${ }^{1} \mathbb{D}$ \\ 1 Instituto Tecnológico de Aragón-ITAINNOVA, C/María de Luna 7-8, 50018 Zaragoza, Spain; \\ vzambrano@itainnova.es (V.Z.); lgracia@itainnova.es (L.A.G.); iviejo@itainnova.es (I.V.); \\ sizquierdo@itainnova.es (S.I.); jrvaldes@itainnova.es (J.R.V.) \\ 2 Institut für Dynamik und Schwingungen—IDS, Leibniz Universität Hannover-LUH, An der Universität 1, \\ 30823 Garbsen, Germany; brase@ids.uni-hannover.de (M.B.); wangenheim@ids.uni-hannover.de (M.W.) \\ * Correspondence: bhernandez@itainnova.es
}

check for updates

Citation: Zambrano, V.; Brase, M.;

Hernández-Gascón, B.;

Wangenheim, M.; Gracia, L.A.;

Viejo, I.; Izquierdo, S.; Valdés, J.R. A

Digital Twin for Friction Prediction in

Dynamic Rubber Applications with

Surface Textures. Lubricants 2021, 9,

57. https://doi.org/10.3390/

lubricants 9050057

Received: 30 March 2021

Accepted: 16 May 2021

Published: 20 May 2021

Publisher's Note: MDPI stays neutral with regard to jurisdictional claims in published maps and institutional affiliations.

Copyright: (c) 2021 by the authors. Licensee MDPI, Basel, Switzerland. This article is an open access article distributed under the terms and conditions of the Creative Commons Attribution (CC BY) license (https:/ / creativecommons.org/licenses/by/ $4.0 /)$.
Abstract: Surface texturing is an effective method to reduce friction without the need to change materials. In this study, surface textures were transferred to rubber samples in the form of dimples, using a novel laser surface texturing (LST) — based texturing during moulding (TDM) production process, developed within the European Project MouldTex. The rubber samples were used to experimentally determine texture-induced friction variations, although, due to the complexity of manufacturing, only a limited amount was available. The tribological friction measurements were hence combined with an artificial intelligence (AI) technique, i.e., Reduced Order Modelling (ROM). ROM allows obtaining a virtual representation of reality through a set of numerical strategies for problem simplification. The ROM model was created to predict the friction outcome under different operating conditions and to find optimised dimple parameters, i.e., depth, diameter and distance, for friction reduction. Moreover, the ROM model was used to evaluate the impact on friction when manufacturing deviations on dimple dimensions were observed. These results enable industrial producers to improve the quality of their products by finding optimised textures and controlling nominal surface texture tolerances prior to the rubber components production.

Keywords: reduced order modelling; dynamic friction; rubber seal applications; tensor decomposition; laser surface texturing; texturing during moulding; digital twin; machine learning; artificial intelligence

\section{Introduction}

\subsection{Surface Texturing in Tribological Applications}

In most dynamic rubber applications, low friction is desired to increase the energy efficiency of the tribological system. Therefore, improved friction behaviour is an important design objective in the development process of components like dynamic seals. Within the industrial and scientific communities, significant improvements in dynamic seal performance have been achieved through a series of technological advances, such as the introduction of low-friction polymers [1]. However, this approach is limited by the operating conditions, such as temperatures or the chemical resistance of the new materials.

Surface texturing is an effective method to modify the friction level without changing materials or the lubricant in the dynamic seal contact. An early method of surface texturing, used to improve the tribological performance of mechanical components, is cylinder honing in internal-combustion engines [2]. Further experimental and theoretical studies revealed a significant reduction in friction due to grooves [3] and, in particular, micro dimples in the reciprocating contact of piston rings and cylinder bores of combustion engines $[4,5]$.

The analysis of laser surface textured (LST) piston rings have been extended to modelbased and experimental investigations of mechanical seals, identifying optimised dimple 
dimensions with lowest friction for specific operating conditions [6,7]. In this context, the dimples are advantageous as they serve as hydrodynamic bearings and exhibit the ability to store lubricant [8]. Moreover, the surface textures reduce the real area of contact and trap wear particles $[9,10]$.

Other authors even highlighted the positive effect of surface textured rubber seals in soft elasto-hydrodynamic lubrication (SEHL), resulting in a significant friction reduction compared to the untextured references [11-13]. However, it has also been found that an inappropriate selection of the surface texture dimensions leads to a detrimental increase in friction due to excessive enlargement of cavitation zones in the lubricant, causing a reduced local film thickness and load carrying capacity [14,15]. Furthermore, the influence of the real dimple shape of textured mechanical seals has been identified as an important factor that needs to be considered for valid friction determination [16].

\subsection{Reduced Order Modelling}

The introduction of extended methods, based on artificial intelligence (AI), which go further than usual tribological measurements, is beneficial for friction predictions. The selected AI technique is based on the digital twin (DT) paradigm, i.e., a virtual representation of reality [17-19]. Several techniques belong within the DT paradigm, the most popular being Reduced Order Modelling (ROM) [20,21] and machine learning (ML) [22]. Both techniques are based on mathematical models for real-time simulations. In particular, ROM consists of a set of numerical strategies for multi-variable problem simplification to solve complex numerical systems and it aims to describe and, hence, predict a system's behaviour through a mathematical approximation, by preserving its main characteristics, as described in [23]. Tensor rank decomposition (TRD) approach is a non-intrusive, i.e., completely data-driven, method that allows to describe a complex system's behaviour, where its variables influence each other, as a simplified mathematical function that describes each variable's effect separately. As previously detailed in [23], TRD is based on the assumption that a problem of $\mathrm{N}$, not necessarily independent, variables can be rewritten as the product of $\mathrm{N}$ one-dimensional functions, one for each of the variables of the system, as shown in Equation (1):

$$
F\left(v_{1}, \ldots, v_{N}\right)=\sum_{m=1}^{M} \alpha_{m} \prod_{n=1}^{N} f_{m, n}\left(v_{n}\right)
$$

where $M$ is the order of approximation of the ROM model and $\alpha_{m}, m=1, \ldots, M$ are weighting coefficients. The functions $f_{m, n}$, in their most simple form, are piecewise linear functions; hence the adjustment parameters are the positions and the values at which the functions change slope. The adjustment of all these parameters is carried out through a least square optimisation.

The first term of the sum in Equation (1) represents a first approximation of the system, being its corresponding coefficient $\alpha_{1}$ the largest one, while the following terms would be corrections to it and will generally have lower coefficient values unless the correction only applies to a specific outliers population and does not affect the general trend of data.

\subsection{Objectives}

Although many experimental and model-based studies have been accomplished on the subject of friction reduction of surface textured components, all investigations require extensive series of experiments or complex contact- or fluid-mechanic simulations. Therefore, this paper aims to introduce a novel approach, in which a limited number of friction measurements of surface textured rubber samples are combined with ROM to identify optimal surface textures as a function of the prevailing operating conditions in real-time. Besides the nominal dimple texture parameters, the real dimple dimensions, defined by diameter, distance, and depth, are taken into account for the friction values 
computed by the ROM. Therefore, ROM technique is further used to determine nominal surface texture values uncertainties for valid friction prediction.

\section{Materials and Methods}

The following Sections are dedicated to the description of the experimental studies and the used software, data analysis (dataset publicly available under [24]), and statistical tools.

\subsection{Rubber Specimen Geometry and Surface Texture Parameters}

The objective of the experimental testing procedure is to measure the friction between surface textured rubber specimens and a rotating counter surface by utilising a pin-on-disc tribometer. The grinded steel counter surface exhibits a surface roughness of $R_{a}=0.50 \mu \mathrm{m}$, but no further texturing. Due to the variety of seal geometries available on the market, a simplified rubber sample geometry was chosen for the experiments, in order to test the surface texture-induced friction variation independent of a specific seal geometry. The corresponding geometry of the rubber samples is shown in Figure 1a.

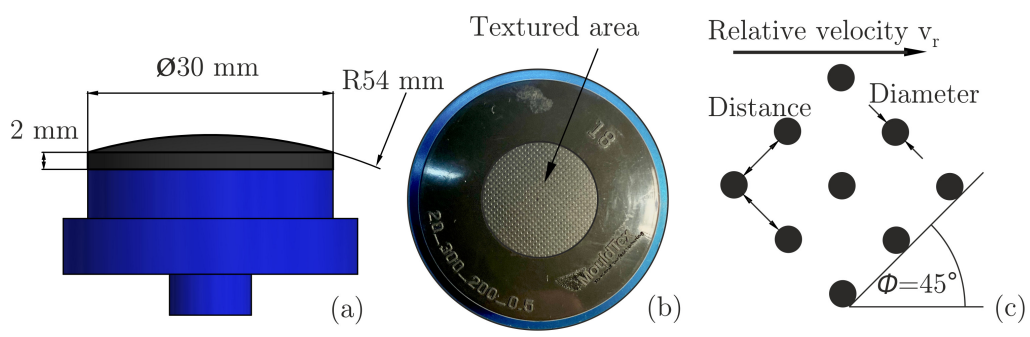

Figure 1. (a) Geometry of the rubber specimens including the most relevant dimensions, (b) picture of the rubber specimen with focus on the dimple texture and (c) positioning of the dimples in relation to the relative velocity vector.

A $2 \mathrm{~mm}$ thick layer of a fluorelastomer with a shore hardness of 80A (FKM 80A) is vulcanised onto a blue anodised aluminium specimen holder. Furthermore, the contact zone of the $30 \mathrm{~mm}$ diameter rubber sample has a spherical shape to avoid edge effects in the dynamic contact.

The surface textures are applied to the contact areas of the rubber samples in the form of deterministic positioned dimples, see Figure $1 \mathrm{~b}$. The geometry of the circular dimples is defined by the diameter, the distance and the depth. The corresponding parameters and the alignment of the dimples are shown in Figure 1c. During the test procedure, the positioning of the dimples, within a squared area, was rotated by an angle of $\phi=45^{\circ}$, since preliminary experiments showed about $20 \%$ greater friction reduction in this arrangement compared to $\phi=0^{\circ}$. The reason for this is the avoidance of continuous flow channels without dimples in the direction of relative motion for $\phi=45^{\circ}$. The angle $\phi$ between the square shaped texture arrangement and the relative velocity vector is visualised in Figure 1c.

In order to perform the experiments, test specimens with eight different surface textures were manufactured by texturing during moulding (TDM). The associated sample number $i$ and the nominal texture parameters are listed in Table 1.

Table 1. Nominal dimple texture parameters defined by diameter, distance and depth.

\begin{tabular}{cccc}
\hline Sample $\boldsymbol{i}$ & $\begin{array}{c}\text { Dimple Diameter } \\
{[\mu \mathrm{m}]}\end{array}$ & $\begin{array}{c}\text { Dimple Distance } \\
{[\mu \mathrm{m}]}\end{array}$ & $\begin{array}{c}\text { Dimple Depth } \\
{[\mu \mathrm{m}]}\end{array}$ \\
\hline 1 & 100 & 300 & 10 \\
2 & 200 & 100 & 10 \\
3 & 200 & 200 & 10 \\
4 & 300 & 100 & 30 \\
5 & 300 & 200 & 20 \\
6 & 300 & 200 & 30 \\
7 & 300 & 300 & 20 \\
8 & - & - & - \\
\hline
\end{tabular}


The parameter range of the dimple dimensions was selected to ensure a transferability of the textures to dynamic seal applications. For example, if the diameter of a dimple is larger than the contact width of a seal, the system would leak. Since the contact width of many lip seals is only $0.80 \mathrm{~mm}$ or less [25], the maximum size of the dimple diameter and distance was set to $300 \mu \mathrm{m}$. In contrast, e.g., the lower limit of the dimple diameter of $100 \mu \mathrm{m}$ was determined by the accuracy of the LST process of the TDM manufacturing method. Based on the texturing results of the rubber samples, the transfer of the textures to real seals has already been successfully realised, but will not be discussed within the scope of this work.

The design of experiment (DoE) on the variation of the surface texture parameters is chosen according to the requirements of the ROM, described in Section 2.4. While seven specimens exhibit a dimple texture, one specimen was produced without dimples to serve as a reference for the dimple-induced friction variation of the other textures. The surface roughness of all eight samples was adjusted to an identical value of $\mathrm{R}_{a}=0.50 \mu \mathrm{m}$ by laser surface processing of the mould. Therefore, the influence of dimple textures on friction is investigated independently of different surface roughnesses.

\subsection{Test Rig Setup and Experimental Procedure for Determining the Coefficient of Friction}

A pin-on-disc tribometer is utilised to measure the friction forces between the rubber specimens and the rotating steel counter surface. The design of the test rig is shown in Figure 2a.

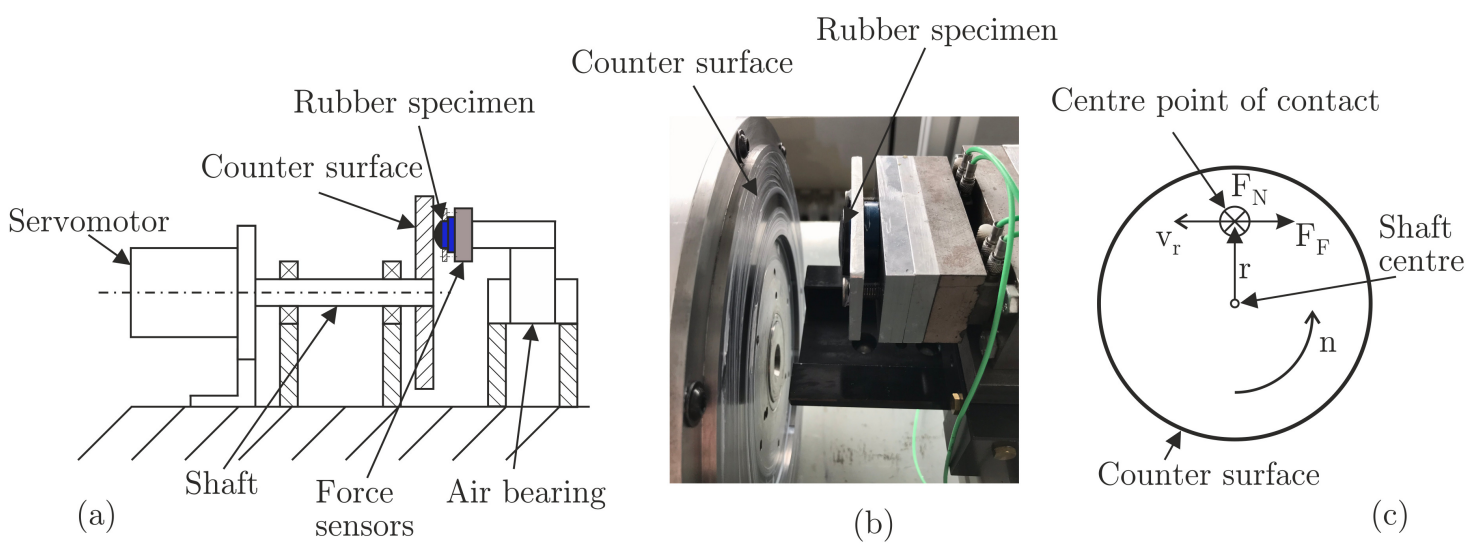

Figure 2. (a) Pin-on-disc tribometer design, (b) picture of the tribometer with focus on the rubber specimen and the rotating counter surface, and (c) contact conditions between the rubber sample and the counter surface.

The rotation of the counter surface is realised by a servomotor. The force sensors measure both the force $F_{N}$ in the normal direction of the sample, as well as the friction force $F_{F}$ in the circumferential direction of the rotating counter surface, compare Figure 2c. The rotational speed $n$ of the counter surface and the normal force $F_{N}$ are kept constant during each measurement, in order to measure quasi-stationary friction values under steady operating conditions. Each relative velocity $v_{r}$, specified in Table $2 \mathrm{a}$, is tested at each of the 3 different contact pressure levels $p_{c, \max }$, given in Table $2 \mathrm{~b}$.

The relative velocity $v_{r}$ between the rubber specimen and the counter surface is equal to the circumferential velocity $v_{c}$ at the point of contact: $v_{r}=v_{c}$. The velocity $v_{r}$ is calculated from the rotational speed $n$ of the counter surface and the distance $r=100 \mathrm{~mm}$ between the shaft centre and the contact point, see Figure 2c, as defined in Equation (2).

$$
v_{r}=\omega r=2 \pi n r
$$

The focus of this paper is on dynamic friction, so static friction is not investigated. Thus, the rotational speed $n$ of the servomotor was varied between 0.6 and $24.0 \mathrm{~min}^{-1}$, resulting in relative velocities $v_{r}$ of 6 to $251 \mathrm{~mm} / \mathrm{s}$. 
Table 2. Operating parameters that are examined during the test procedure. (a) Rotational speeds $n$ of the servomotor and corresponding relative velocities $v_{r}$ between the rubber sample and the counter surface (2), (b) together with the normal force $F_{N}$, the related maximum contact pressure $p_{c, \text { max }}$, the contact diameter $d_{c}$ between the rubber sample and the counter surface, as well as the nominal contact area $A_{\text {nominal }}(2)$.

a

\begin{tabular}{|c|c|c|c|}
\hline \multicolumn{2}{|c|}{$\begin{array}{l}\text { Rotational Speed } \\
\quad n\left[\mathrm{~min}^{-1}\right]\end{array}$} & \multicolumn{2}{|c|}{$\begin{array}{c}\text { Relative Velocity } \\
v_{r}[\mathrm{~mm} / \mathrm{s}]\end{array}$} \\
\hline \multicolumn{2}{|c|}{0.6} & \multicolumn{2}{|c|}{6} \\
\hline \multicolumn{2}{|c|}{1.2} & \multicolumn{2}{|c|}{12} \\
\hline \multicolumn{2}{|c|}{1.8} & \multicolumn{2}{|c|}{19} \\
\hline \multicolumn{2}{|c|}{3.0} & \multicolumn{2}{|c|}{31} \\
\hline \multicolumn{2}{|c|}{6.0} & \multicolumn{2}{|c|}{63} \\
\hline \multicolumn{2}{|c|}{12.0} & \multicolumn{2}{|c|}{126} \\
\hline \multicolumn{2}{|c|}{18.0} & \multicolumn{2}{|c|}{188} \\
\hline \multicolumn{2}{|c|}{24.0} & \multicolumn{2}{|c|}{251} \\
\hline \multicolumn{4}{|c|}{$\mathbf{b}$} \\
\hline $\begin{array}{l}\text { Normal Force } \\
\qquad F_{N}[\mathrm{~N}]\end{array}$ & $\begin{array}{c}\text { Max Contact Pressure } \\
p_{c, \max }[\mathrm{MPa}]\end{array}$ & $\begin{array}{c}\text { Contact Diameter } \\
d_{c}[\mathrm{~mm}]\end{array}$ & $\begin{array}{c}\text { Nominal Contact Area } \\
A_{\text {nominal }}\left[\mathrm{mm}^{2}\right]\end{array}$ \\
\hline 3.9 & 0.5 & 4.2 & 13.8 \\
\hline 7.9 & 0.7 & 5.0 & 19.6 \\
\hline 13.3 & 0.9 & 5.8 & 26.4 \\
\hline
\end{tabular}

The normal forces $F_{N}$ were selected to achieve maximum contact pressures $p_{c, \max }$ of $0.5,0.7$, and $0.9 \mathrm{MPa}$, which are typical values in pneumatic seal applications [26]. Because of the spherical shape of the rubber specimen, the variation of the normal force $F_{N}$ influences not only the magnitude of the parabolic contact pressure distribution $p_{c}$, but also the dimensions of the nominal contact area between the rubber sample and counter surface, see Figure $3 b$. Therefore, not only the maximum of the contact pressure distribution $p_{c, \text { max }}$ is specified in Table $2 \mathrm{~b}$, but also the corresponding contact diameter $d_{c}$ and the respective circular nominal contact area $A_{\text {nominal }}$. The contact pressure distribution $p_{c}$ and the contact diameter $d_{c}$ are computed by finite element analyses, taking into account the rubber thickness of $2 \mathrm{~mm}$. Within the static simulations, the rubber sample is pressed against the counter surface with the defined normal force $F_{N}$, see Figure $3 a$. At this, the coloured spherical contact area of the rubber specimen is brought into contact with the grey flat counter surface, resulting in the specified contact area and contact pressure $p_{c}$.

All components are modelled as 2D axisymmetric parts. A hyper elastic MooneyRivlin material behaviour is assigned to the $2 \mathrm{~mm}$ layer of the FKM80A rubber material, which is specified by the temperature-dependent material parameters $C_{10}=1,442,425.12$, $C_{01}=208,308.34, D=6.059933161 \times 10^{-10}$, considering a Poisson's Ratio of 0.4995 . For both measurements and simulations, the temperature is equal to $20^{\circ} \mathrm{C}$. The material of the anodised aluminium sample holder is modelled as pure elastic part with a Young's modulus of $70 \mathrm{GPa}$ and a Poisson's Ratio of 0.34 . The counter surface is defined as rigid part.

All experiments are performed with an adherent silicone grease OKS 1155 in the dynamic contact, which exhibits a base oil viscosity of $100 \mathrm{~mm}^{2} / \mathrm{s}$ at $25^{\circ} \mathrm{C}$ [27]. The same lubrication and conditioning procedure was applied to every rubber specimen before the actual friction measurements to ensure comparability between the results. Every single measurement lasts for $5 \mathrm{~s}$ and each measurement was repeated 5 times to ensure a statistical certainty. In order to generate the quasi-stationary friction values $\mu_{\text {untext }}$ for rubber specimen 8 and $\mu_{t e x t, i}$ for rubber samples $i=1-7$, the mean value of each measurement is calculated over the entire measuring period of $5 \mathrm{~s}$. The friction coefficients $\mu_{\text {untext }}$ and $\mu_{t e x t, i}$ are evaluated to identify dimple textures with the highest friction reduction potential. In addition, the results are further processed to generate the ROM model. 


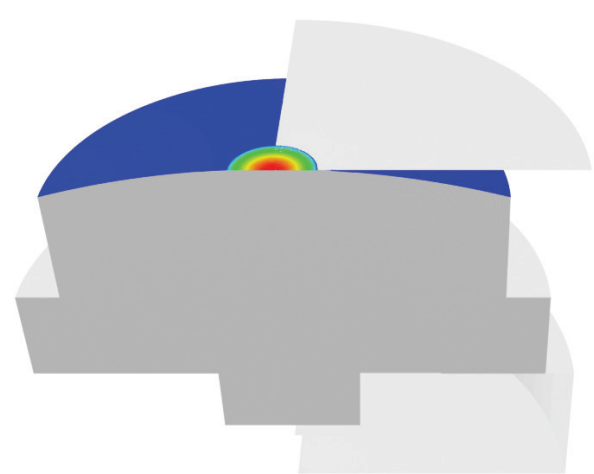

(a)

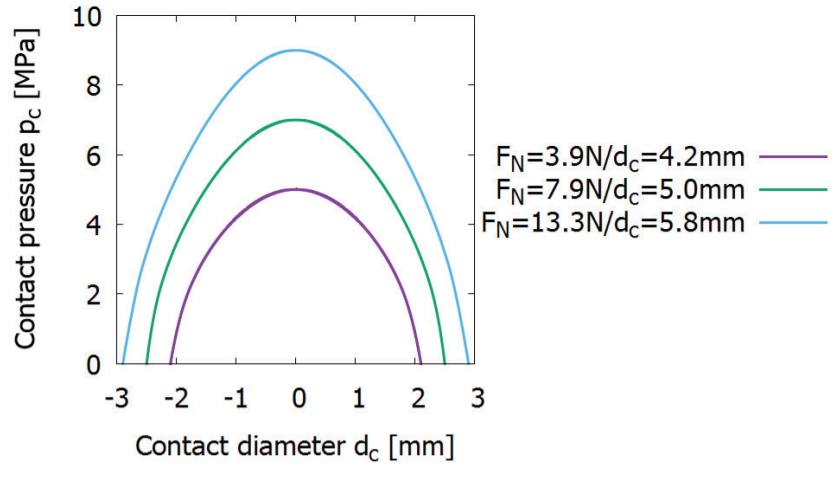

(b)

Figure 3. (a) Assembly of the finite element method (FEM). The coloured spherical contact area of the rubber specimen is brought into contact with the grey flat counter surface. (b) Parabolic contact pressure distribution $p_{c}$ as a function of the contact diameter $d_{c}$.

\subsection{Method of Measurement for Real Dimple Dimensions}

An additional important aspect for the determination of the influence of the dimple textures on friction are the real dimensions of the dimples. While the nominal values of the investigated textures are given in Table 1, the real values of diameter, distance and depth vary due to imperfections in the innovative industrialised TDM production process of the rubber specimens. During this process, the negative of the desired texture is applied to a mould by LST ablation. A 5-axis $100 \mathrm{~W}$ pico-second laser is utilised for this purpose. During the injection moulding and vulcanisation procedure, the texture is directly transferred from the mould to the rubber surface. Protrusions in the mould become dimples in the elastomer. Due to imperfections in the laser machining of the mould, both a deviation from the nominal dimple parameters and ring-shaped cavities in the peripheral areas of the dimple valleys are identifiable, see Figure $4 \mathrm{~b}$. These deviations mainly result from re-solidifying metal vapours on the surface of the metallic vulcanisation mould.

In order to measure and visualise the actual dimple texture dimensions, a 3D optical microscope, based on focus-variation, is used. Figure 4 a shows an exemplary $4 \times 4 \mathrm{~mm}$ surface scan of rubber specimen 6 with a nominal dimple diameter of $300 \mu \mathrm{m}$, a distance of $200 \mu \mathrm{m}$ and a depth of $30 \mu \mathrm{m}$.

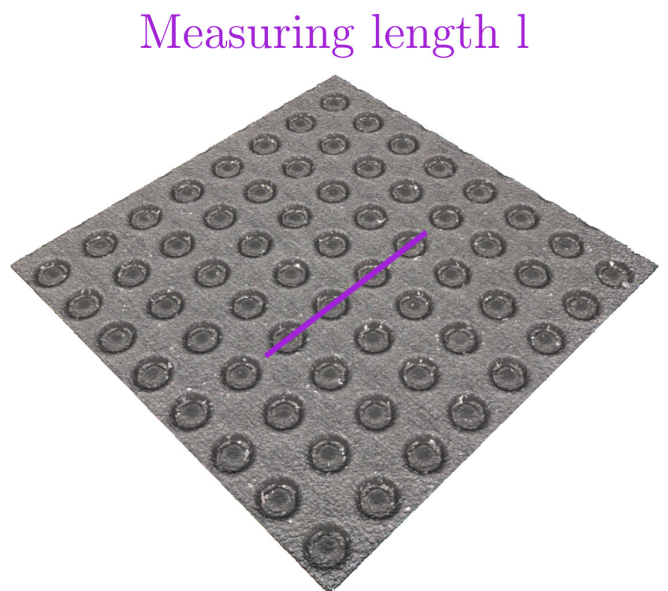

(a)

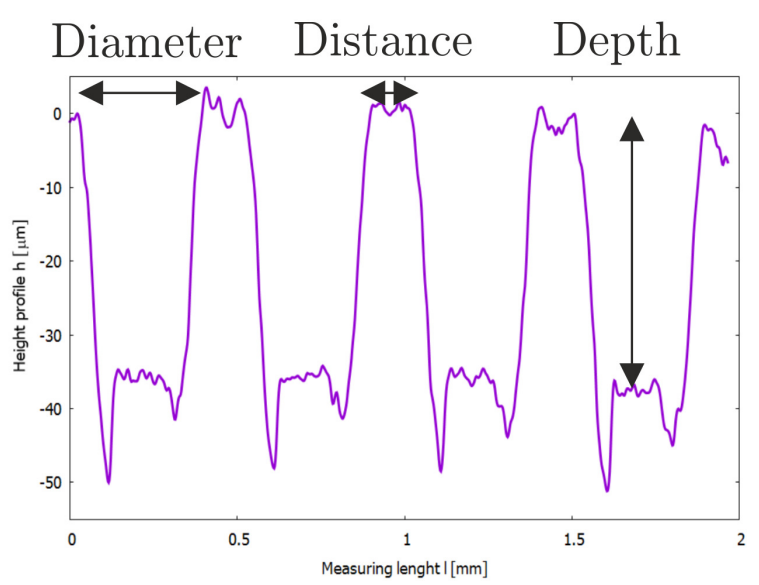

(b)

Figure 4. (a) Microscope image of rubber specimen 6, considering a measured area of $4 \times 4 \mathrm{~mm}$ and (b) related height profile $h$ as function of the measuring length $l$, considering the indicated line scan trough four adjacent dimples.

Figure $4 \mathrm{~b}$ shows the corresponding line scan as height profile $h$ over the measuring length $l$, which is measured through the centre of four adjacent dimples. The positions that 
are measured to determine the real dimple dimensions of diameter, distance, and depth are marked in the height profile. To ensure accurate measurement results of the dimple dimensions, the curvature of the contact surface of the rubber samples was subtracted, resulting in the flat surface scan depicted. Beyond this, no corrections were made to the surface scans. The dimple dimensions were measured at 16 different positions of the textured area of each rubber sample. The mean values of the 16 measurements and the corresponding dimensionless texture parameters are listed in Table 3.

Table 3. Real dimple dimensions, indicated by diameter, distance, and depth. The aspect ratio is the quotient of dimple depth and diameter. The textured area percentage is determined from the quotient of the textured area $A_{\text {textured }}$ and the nominal contact area $A_{\text {nominal }}$.

\begin{tabular}{|c|c|c|c|c|c|}
\hline Sample $i$ & $\begin{array}{l}\text { Dimple Diameter } \\
{[\mu \mathrm{m}]}\end{array}$ & $\begin{array}{c}\text { Dimple Distance } \\
{[\mu \mathrm{m}]}\end{array}$ & $\begin{array}{c}\text { Dimple Depth } \\
{[\mu \mathrm{m}]}\end{array}$ & Aspect Ratio & $\begin{array}{c}\text { Textured Area } \\
{[\%]}\end{array}$ \\
\hline 1 & 135 & 258 & 16 & 0.11 & 9 \\
\hline 2 & 242 & 100 & 11 & 0.05 & 39 \\
\hline 3 & 241 & 165 & 10 & 0.04 & 28 \\
\hline 4 & 337 & 66 & 35 & 0.10 & 55 \\
\hline 5 & 330 & 170 & 22 & 0.06 & 34 \\
\hline 6 & 346 & 153 & 35 & 0.10 & 37 \\
\hline 7 & 336 & 252 & 20 & 0.06 & 25 \\
\hline 8 & - & - & - & - & - \\
\hline
\end{tabular}

\subsection{Software Development for Reduced Order Modelling}

The software library (Twinkle), implemented and used to compute the ROM models, is available on GitHub platform [28] and described in [23], where its basic concept, structure and environmental dependencies are detailed.

Within this study, two separate ROM models were computed: one for untextured and one for textured tribometer samples data, consisting of 24 and 168 data points, respectively. The reliability of the two obtained ROM models was then validated using Python Scikitlearn [29] (version 0.22.1) $\mathrm{k}$-fold cross validation technique, where a train-test procedure is performed k-times, randomly extracting a k-fraction sub-dataset for testing [30]. For the validation, the parameter $k$ was set to 10 , so that 10 different train-test validations were performed, randomly selecting the corresponding $90 \%-10 \%$ data fractions each time a new validation was performed.

Moreover, minimum values of the ROM function, i.e., Equation (1), were obtained using the ALGLIB Free Edition library [31], version 3.14.0, to find the surface texture dimensions that allow minimising friction, as described in Section 3.5.

Twinkle library can be described through the supervised learning algorithm concept in ML [32,33]. The input values used for ROM construction were the dimple dimensions, i.e., depth, diameter and distance, when available, together with pressure and velocity (please refer to Tables 1 and 2 or the available dataset under [24]), the output being the experimentally measured friction coefficient. Within the scope of this paper Equation (1) would represent the friction coefficient, expressed as the sum of 2 or 17 terms (in case of untextured or textured surface data, respectively). Each term being the product of one-dimensional functions, i.e., one function for each input separately, as shown for simplicity in Equation (3) for the untextured case.

$$
\left.\left.\mu(\text { pressure }, \text { velocity })=\alpha_{1}\left[f_{1}(\text { pressure }) f_{1} \text { (velocity }\right)\right]+\alpha_{2}\left[f_{2}(\text { pressure }) f_{2} \text { (velocity }\right)\right]
$$

where $\alpha_{1}$ and $\alpha_{2}$ are the weighting coefficients of each computed term. The functions $f_{1}$ and $f_{2}$ are one-dimensional piecewise linear functions that describe the impact, on the friction outcome, of each input separately. 


\subsection{Reduced Order Modelling Data Pre-Processing for Friction Reduction}

The friction variations (in\%) obtained with the introduction of a dimple texture on the rubber surface are evaluated using Equation (4) in order to quantify the surface textureinduced friction variations.

$$
\Delta \mu=100 \frac{\mu_{\text {untext }}-\mu_{\text {text }, i}}{\mu_{\text {untext }}} \%
$$

In Equation (4) textured cases are compared to their corresponding untextured result, so that a negative value suggests a friction increase and a positive one reveals a friction reduction.

The final $\Delta \mu$ prediction was obtained using Equation (4), where the $\mu_{\text {untext }}$ and the $\mu_{\text {text }, i}$ values, predicted by the ROM models, were used, instead of using the experimentally measured results.

The two datasets, prepared and used in the statistical analysis, were created from two data samples of 48 data points each, using Python NumPy version 1.18.1 [34] and SciPy version 1.4.1 [35] libraries, as described in Sections 2.6 and 3.3.

\subsection{Statistical Analysis of Real Dimple Dimensions}

Due to manufacturing tolerances, the real dimple dimensions are not equal to the nominal ones. Experimental data were used to extract statistical information from 16 measurements of geometrical parameters of the textured surface, i.e., mean values and standard deviations for the three geometrical parameters were identified: dimple depth, diameter, and distance. The measuring procedure and mean values are described in Sections 2.3 and 3.1, respectively. By comparing the results to a foreseen normal distribution centred on the nominal value, having as standard deviation the value obtained from the different repetitions, it was possible to infer the type of probability density function (PDF) of each of the studied parameters.

Once the PDFs were known, the dimple depth was varied separately, while diameter and distance were varied together, as their variations are not independent and occur in the same plane, as defined by the textured area (Section 3.1). To do that, not all data were taken into account, but only those dimple parameters whose variations fell within the ROM's definition limits, minimum and maximum values in Tables 1 and 2, which resulted in 48 data points for both datasets, that were obtained when vertical, i.e., depth, and horizontal, i.e., diameter and distance, variations were applied.

The PDFs were hence created by means of a Python script and were used to generate the above mentioned datasets of 48 data points each, as it follows. Each original point was replicated 100 times where the nominal values were changed accordingly to their evaluated PDFs and a final amount of 4800 points were obtained. As mentioned before, the described approach was performed two times: one repeating depth values only and a second time replicating diameter and distance values together. Since dimple diameter and distance are dependent magnitudes, these were made to vary accordingly to one another as follows: at first a new diameter value was randomly generated through the known PDF, then the cumulative density function (CDF) was obtained for the specific diameter value and the corresponding distance value was calculated as the value holding the complement of the CDF in the distance PDF.

The new obtained dataset contains input parameters statistical fluctuations and was used to perform ROM predictions on the enlarged sets of points, where the dimple values experimental variations on dimple dimensions' nominal values are taken into account. The effects on friction due to the experimental texturing deviations were then analysed and a $\mathrm{t}$-Student test was used for a statistical comparison of the obtained results to an ideal PDF around nominal surface texture values, as described in Section 3.4. 


\section{Results}

In the following subsections the results obtained from the experimental tribotests and dimple dimensions nominal values assessment, the data pre-processing and the statistical analysis of textures dimensions are detailed.

\subsection{Measurement Results of the Real Dimple Dimensions and Definition of Dimensionless Dimple Parameters}

The aim of the real dimple dimensions measurement procedure, which has been previously described in Section 2.3, is the identification of the effective texture geometry. While the real dimple diameter and depth reveal larger values compared to the nominal dimensions, the dimple distances are smaller than the nominally specified values, compare Tables 1 and 3.

In addition to the diameter, distance, and depth dimple parameters, the dimensionless aspect ratio and textured area (equal to area density), which are often referred in literature [36-38], are further specified in Table 3 . The aspect ratio is the quotient of the dimple depth and the dimple diameter and varies between 0.04 and 0.11 for the analysed textures. The area density is equal to textured area percentage. It is calculated by the quotient of the textured area $A_{\text {textured }}$ and the nominal circular contact area $A_{\text {nominal }}$, which are both visualised in Figure 5 .
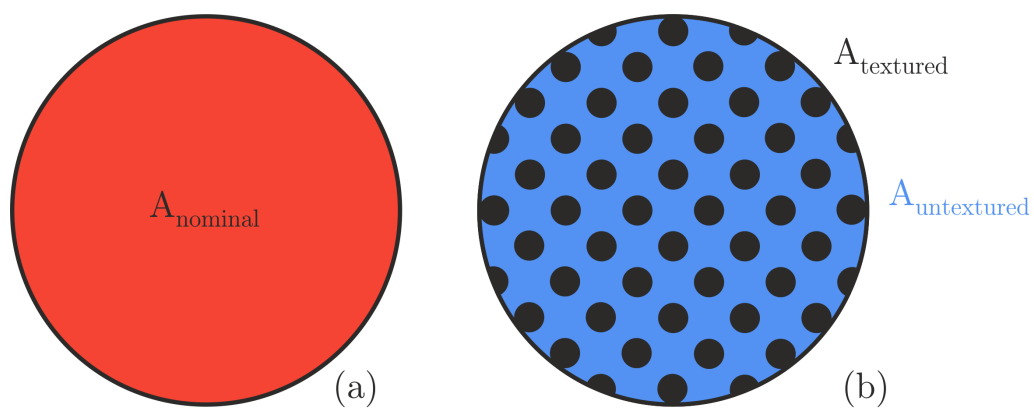

Figure 5. (a) Red marked nominal circular contact area $A_{\text {nominal }}$ between an untextured rubber specimen and the counter surface and $(\mathbf{b})$ contact area of a textured sample. The textured area $A_{\text {textured }}$ is indicated by the black circular dimples, the untextured area that is in direct contact with the counter surface is coloured in blue.

The area density of the studied textures varies between $9 \%$ and $55 \%$. Despite the fact that the three different normal forces $F_{N}$ analysed result in three different nominal contact areas $A_{\text {nominal }}$, the percentage textured area is independent of the nominal contact area $A_{\text {nominal }}$, since more dimples come into contact with increasing normal force $F_{N}$, compare also Table $2 \mathrm{~b}$.

\subsection{Reduced Order Modelling on Friction Coefficient Data}

The two ROM functions (please refer to Equations (1) and (3)), obtained for untextured and textured friction coefficient datasets, converged to a stable solution, using only 2 and 17 terms, respectively. In order to assess the correctness and precision of the ROM results, the predicted value is compared to the corresponding experimental one, as ideally, both should be the same. The prediction lines are plotted, for both untextured and textured ROM models, in Figure 6a,b.

From Figure $6 \mathrm{a}, \mathrm{b}$ it is possible to conclude that the ROM prediction is extremely accurate, being the obtained ROM models' standard deviations $\sigma_{\text {untext }}=0.0014$ and $\sigma_{\text {text }}=0.0012$ for untextured and textured data, respectively, and both regression lines (dashed black) match the goal prediction line (solid red).

Both ROM models were validated using the $\mathrm{k}$-fold cross validation technique (see Section 2.4). The results obtained for the $R^{2}$ are shown in Table $4 \mathrm{a}, \mathrm{b}$ for untextured and textured data, respectively. 


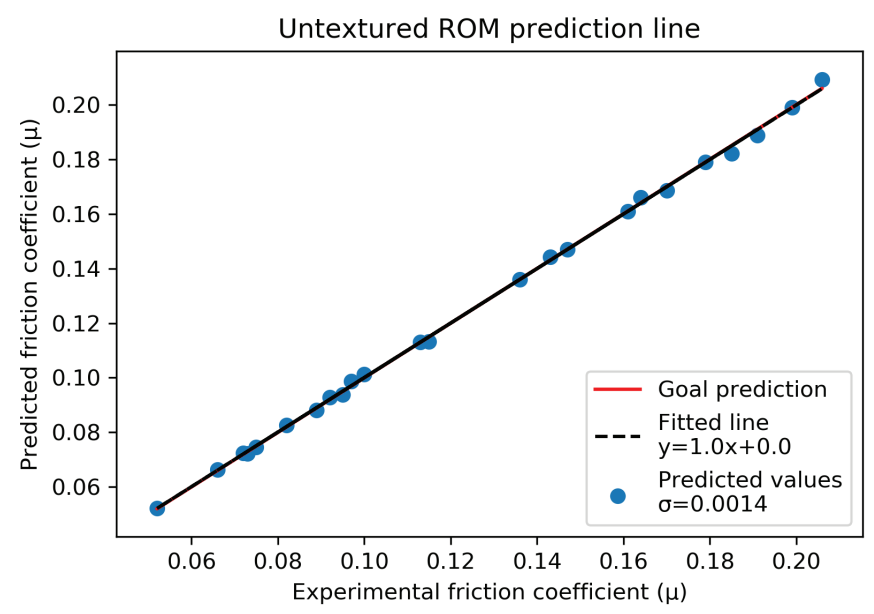

(a)

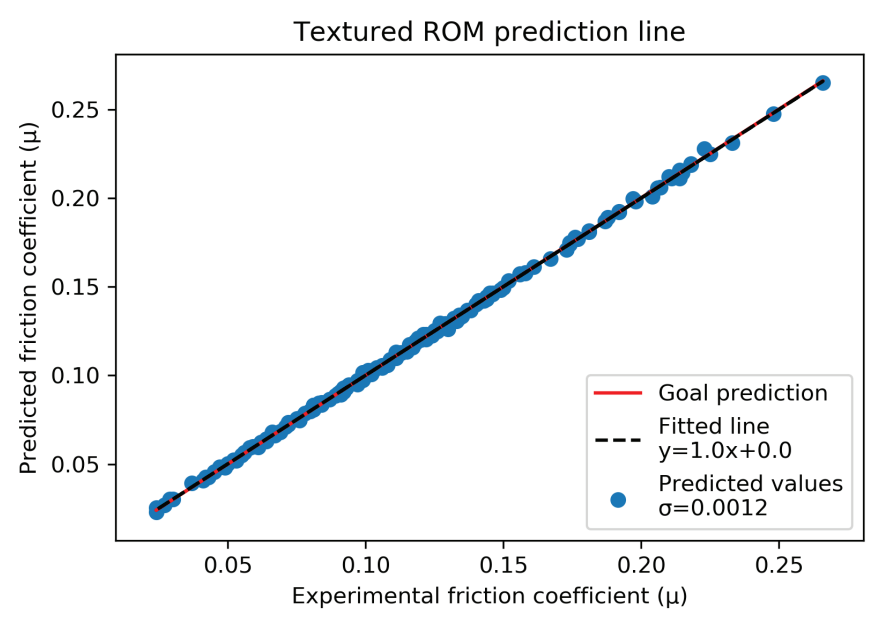

(b)

Figure 6. ROM prediction for (a) untextured and (b) textured friction coefficient data. The blue dots are obtained by evaluating the ROM with the experimental data, the dashed black line is the linear regression that fits the data, while the solid red line represents the ideal ROM result, where the ROM evaluation on each experimental friction value returns the same value.

Table 4. (a) Untextured and (b) Textured ROM $R^{2}$ for Train and Test datasets when the k-fold cross validation method is applied.

\begin{tabular}{|c|c|c|c|c|c|}
\hline \multicolumn{3}{|c|}{ a } & \multicolumn{3}{|c|}{ b } \\
\hline \multicolumn{3}{|c|}{ Untextured ROM $R^{2}$} & \multicolumn{3}{|c|}{ Textured ROM $R^{2}$} \\
\hline & Train & Test & & Train & Test \\
\hline 1 & 1.000 & 0.895 & 1 & 1.000 & 0.914 \\
\hline 2 & 1.000 & 0.968 & 2 & 1.000 & 0.872 \\
\hline 3 & 1.000 & 0.988 & 3 & 1.000 & 0.882 \\
\hline 4 & 1.000 & 0.974 & 4 & 1.000 & 0.943 \\
\hline 5 & 1.000 & 0.988 & 5 & 1.000 & 0.923 \\
\hline 6 & 1.000 & 0.903 & 6 & 1.000 & 0.896 \\
\hline 7 & 1.000 & 0.871 & 7 & 1.000 & 0.963 \\
\hline 8 & 1.000 & 0.994 & 8 & 1.000 & 0.912 \\
\hline 9 & 1.000 & 0.915 & 9 & 1.000 & 0.953 \\
\hline 10 & 1.000 & 0.982 & 10 & 1.000 & 0.938 \\
\hline avg & 1.000 & 0.948 & avg & 1.000 & 0.919 \\
\hline
\end{tabular}

Table $4 a$, b show that for both ROM models the results of the validation are very precise and that there is always an excellent correlation between the input data, i.e., untextured or textured friction coefficient data (available under [24]), and the ROM prediction.

\subsection{Reduced Order Modelling on Pre-Processed Data for Friction Variations}

As described in Section 2.5, the friction variations were computed according to Equation (4), both for experimental and ROM predicted data. Similarly to Section 3.2, a prediction line was obtained to assess the accuracy of the results, obtained as the combination of the two (untextured and textured) ROM models. The results are shown in Figure 7. 


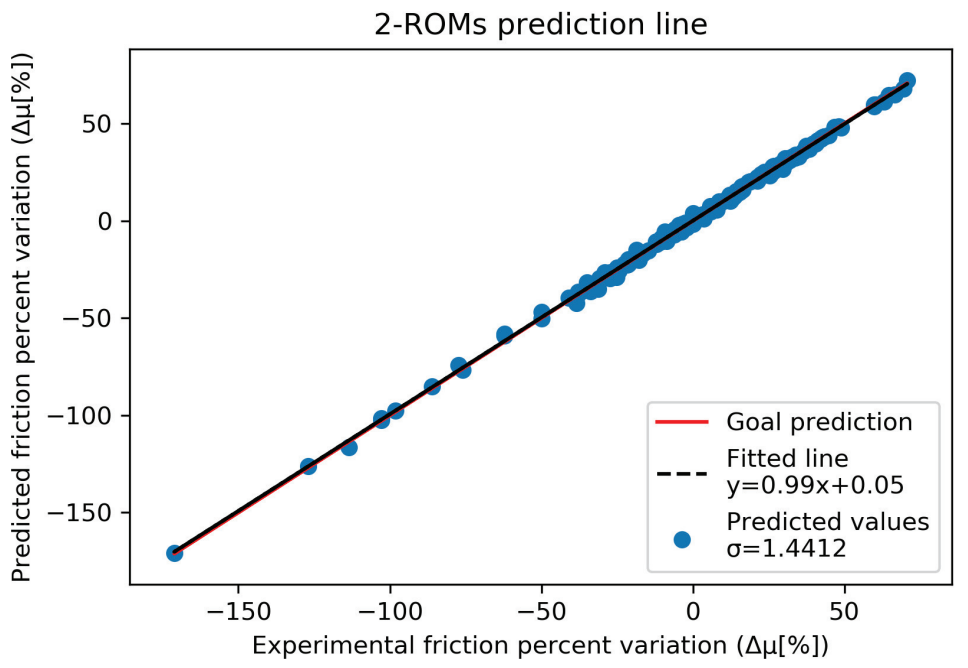

Figure 7. 2-ROM-models prediction for combined untextured and textured friction data, according to Equation (4). The blue dots are obtained by evaluating the combined 2-ROM predictions with the combined experimental data (Equation (4)), the dashed black line is the linear regression that fits the data, while the solid red line represents the ideal prediction result, where the predicted values perfectly match the experimental ones.

From Figure 7 it is observed that the ROM prediction is highly accurate, since the regression line (dashed black) is highly comparable to the goal prediction (solid red).

Moreover, an error propagation was performed on the friction percent variation variable (Equation (4)), in order to assess the maximum error assumed by the predictive model. For this purpose, experimental deviations on friction measurements were not included, since these are not considered intrinsic properties of the prediction accuracy, but depend on the a-priori goodness of the dataset solely. The results obtained when taking into account both the error propagation on $\Delta \mu$ and the standard deviation obtained for the 2-ROM prediction, i.e., $\sigma=1.4412$ as shown in Figure 7, show values between $2 \%$ and $9 \%$, with a mean value of $3 \%$ and $95 \%$ of data with a prediction deviation below $5 \%$. Moreover, a study was performed to check the impact that each input parameter has on the measured friction, according to Equations (1) and (3), as shown in Figure 8 for textured ROM.
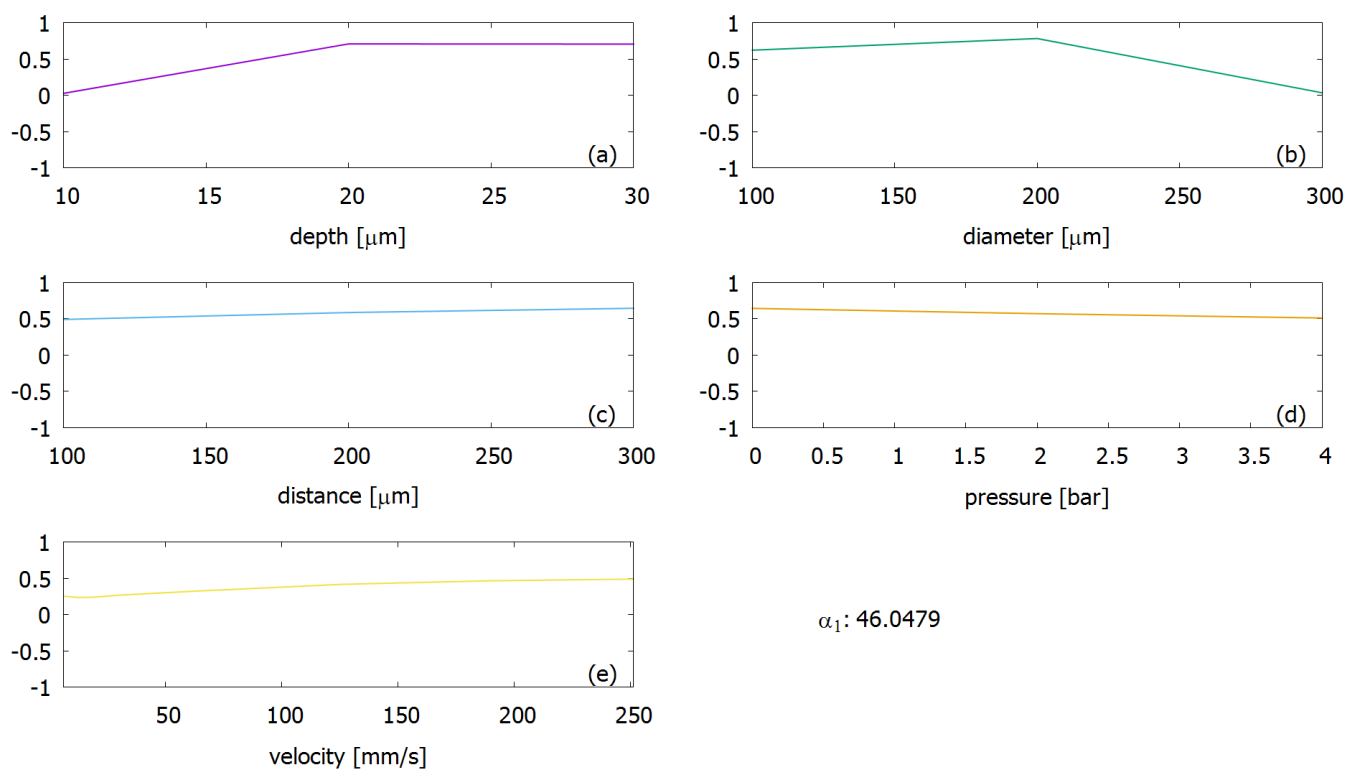

$\alpha_{1}: 46.0479$

Figure 8. Textured data ROM's first term. The one-dimensional functions, for each variable are shown separately in the plots, according to Equation (1), labelled with letters from (a-e). 
The one-dimensional functions (specified in Equation (1)) for the textured ROM, shown in Figure 8, represent the impact of each input on the friction variation. The textured ROM shows $\alpha_{1}=46.0479$ for the first term (see Equations (1) and (3)), being its weighting factor one or two orders of magnitude higher than the remaining terms. The first term of the ROM series expansion can be, hence, considered a fair approximation of the system, where other terms are corrections to it, as described in Section 1.2. From Figure 8 one can see that bigger friction variations occur at extreme surface texturing parameters, e.g., smaller dimple depth and bigger diameter values.

\subsection{Statistical Analysis Results of Real Dimple Dimensions}

As described in Section 2.6, PDFs were extrapolated for all texture parameters and were used to assess the measured differences from the desired nominal dimple values. The observed variations from the nominal dimple dimensions, described in Section 2.3, were introduced into the datasets in order to predict the corresponding friction variation using the previously computed ROM. The observed PDFs were normal distributions for both dimple depth and distance, while for diameter a skewed normal distribution was observed. dimple depth PDF showed a right shift of the mean equal to $3 \mu \mathrm{m}$ and a standard deviation equal to $1.5 \mu \mathrm{m}$. Concerning the distance, the right shift of the mean was equal to $41 \mu \mathrm{m}$ and $37 \mu \mathrm{m}$ for corresponding nominal values of $200 \mu \mathrm{m}$ and $300 \mu \mathrm{m}$, with a standard deviation of $13 \mu \mathrm{m}$ and $9 \mu \mathrm{m}$, respectively. These findings led to significant differences in observed friction values, which means that deviations from nominal texture values, shown in Figures 9 and 10a,b, do actually affect friction as observed in Figure 11a,b.

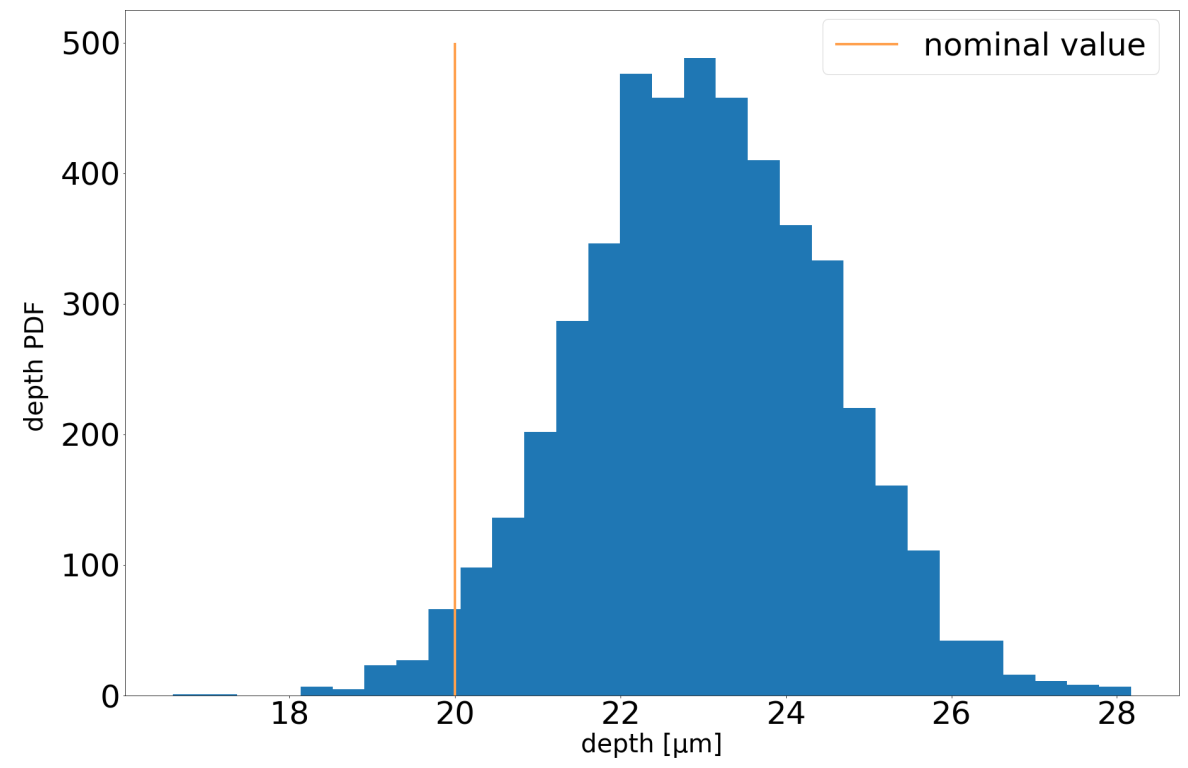

Figure 9. Dimple depth specific PDF and corresponding nominal value.

A ROM model allows verifying that the introduction of a statistical noise on nominal surface texture values affects friction, as shown in Figure 11a,b. From these Figures it is possible to observe significant variations in friction PDFs distributions when depth or diameter and distances, respectively, vary from nominal values, and to compare the obtained friction distribution to a normal PDF with zero mean and $\sigma=0.01$.

A two-tailed t-Student test was performed to check the compatibility of the two PDFs, i.e., the noisy nominal values friction distribution (orange) and the ideal friction distribution (green), shown in Figure 11a,b. The test result showed a statistically significant difference between the noisy and the ideal PDF in case of dimple diameter-distance variations, according to what can be expected from Figures 9 and 10a,b. 


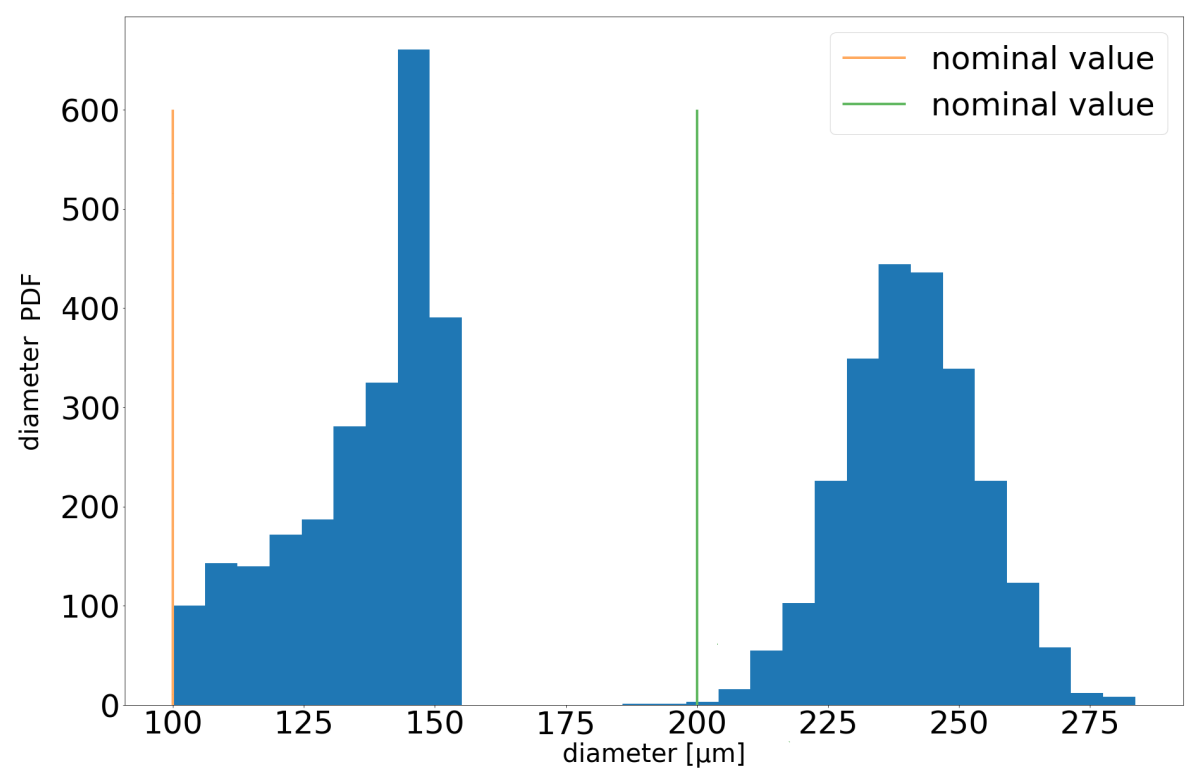

(a)

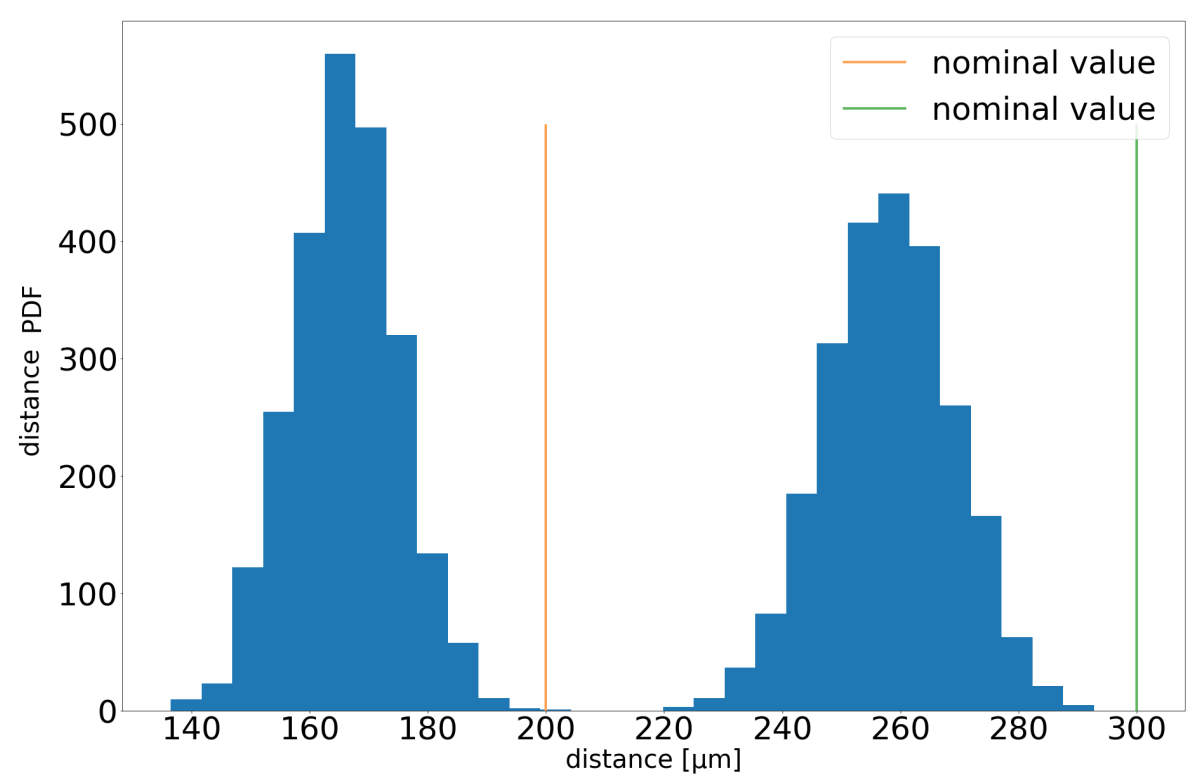

(b)

Figure 10. (a) Dimple diameter and (b) distance specific PDFs and respective nominal values.

As previously described in Section 2.5, two expanded datasets were generated introducing statistical noise to the textured data sample by varying the nominal dimple parameters (i.e., depth, diameter, and distance) according to the obtained PDFs. When depth was made to vary, only values equal to $20 \mu \mathrm{m}$ could be used; in fact, nominal depth values of $10 \mu \mathrm{m}$ and $30 \mu \mathrm{m}$ would cause friction values to fall outside the ROM domain if a PDF was applied to generate data around these values. In this case the corresponding ranges for dimple diameter and distance were $200 \mu \mathrm{m}$ and 200-300 $\mu \mathrm{m}$, respectively. For the second dataset, given the ROM definition domain, feasible dimple diameter and distance ranges resulted in 100-200 $\mu \mathrm{m}$ and 200-300 $\mu \mathrm{m}$, respectively, corresponding to depth values of $10 \mu \mathrm{m}$ only. It is important to remark that, in this case, given the geometrical definition of the dimples, diameter and distance are inversely correlated and that the two PDFs are very different, being the first one a skewed distribution and the second a normal one (please refer to Section 2.5 for method details). 


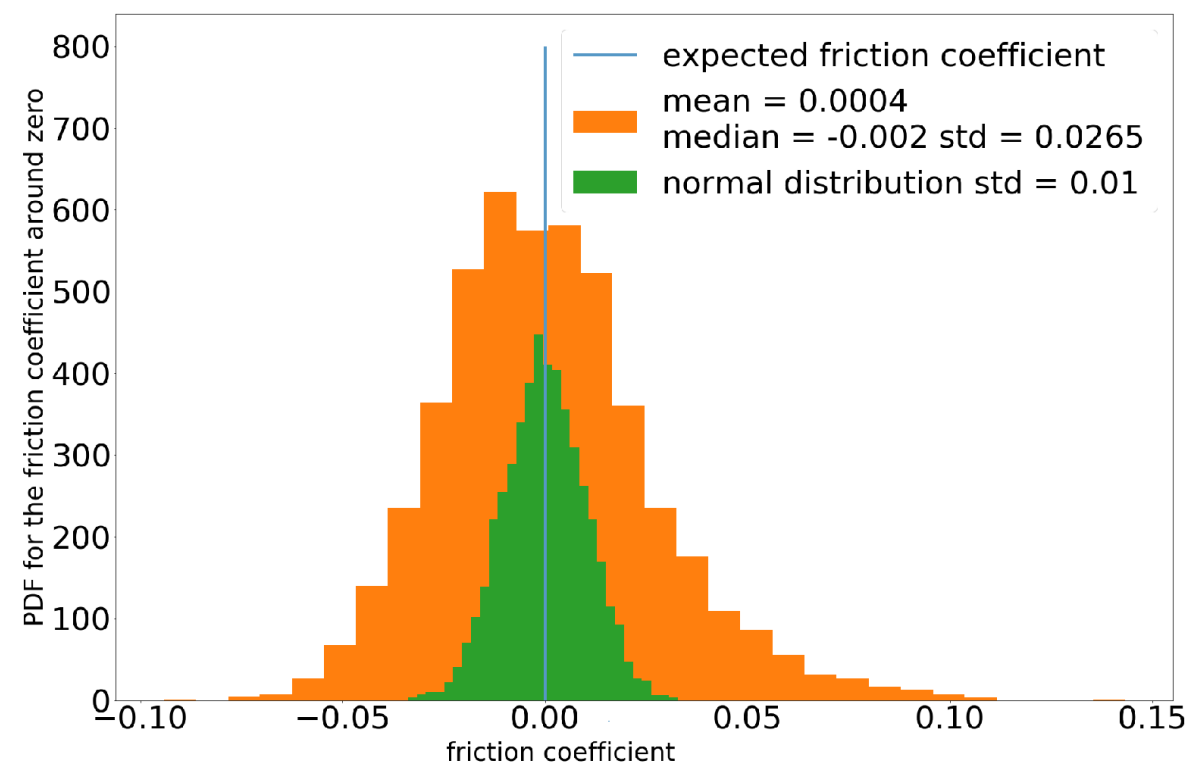

(a)

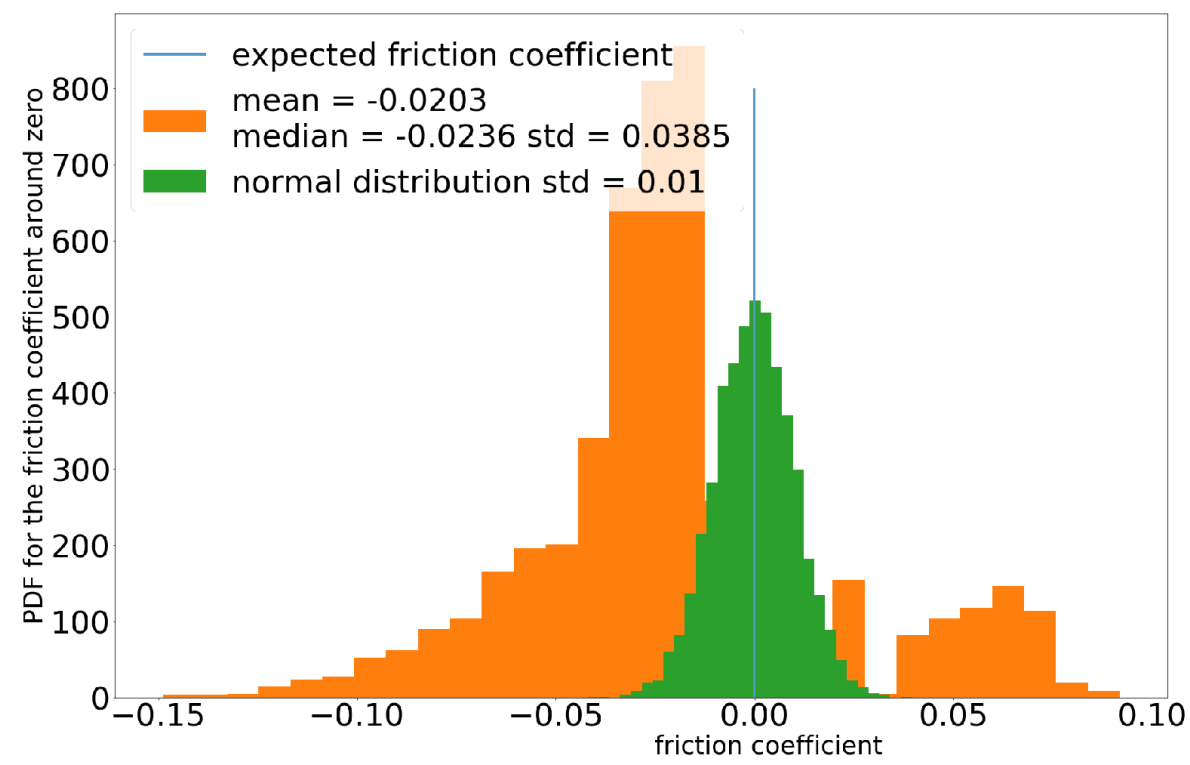

(b)

Figure 11. Friction coefficient distribution (orange) when (a) dimple depth or (b) diameter and distance are varried according to their specific PDFs (Figures 9 and 10a,b, respectively), centred and compared to a normal distribution (green) with zero mean and $\sigma=0.01$.

Figure $12 \mathrm{a}, \mathrm{b}$ show the findings for the friction prediction when the geometrical parameters of the seals deviate from nominal values. 


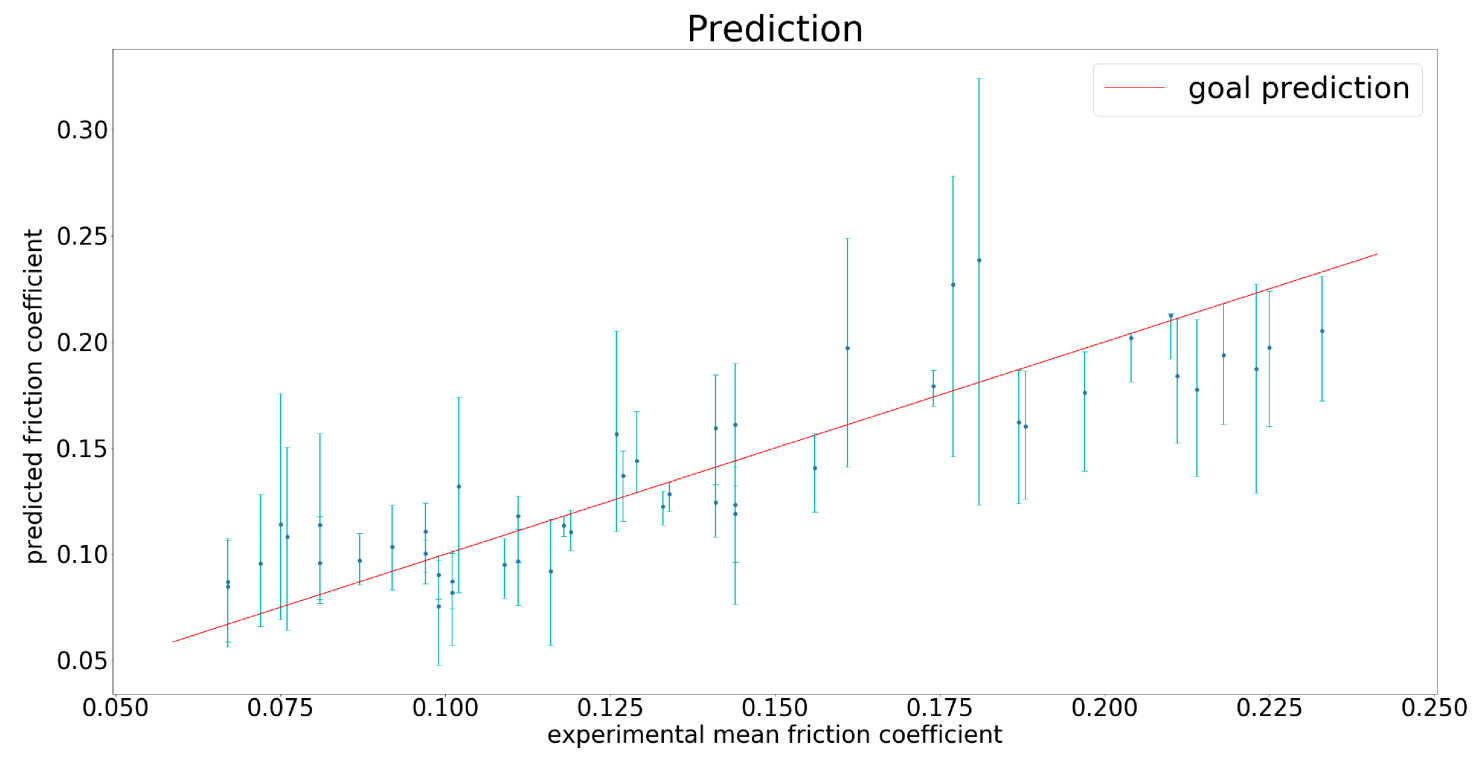

(a)

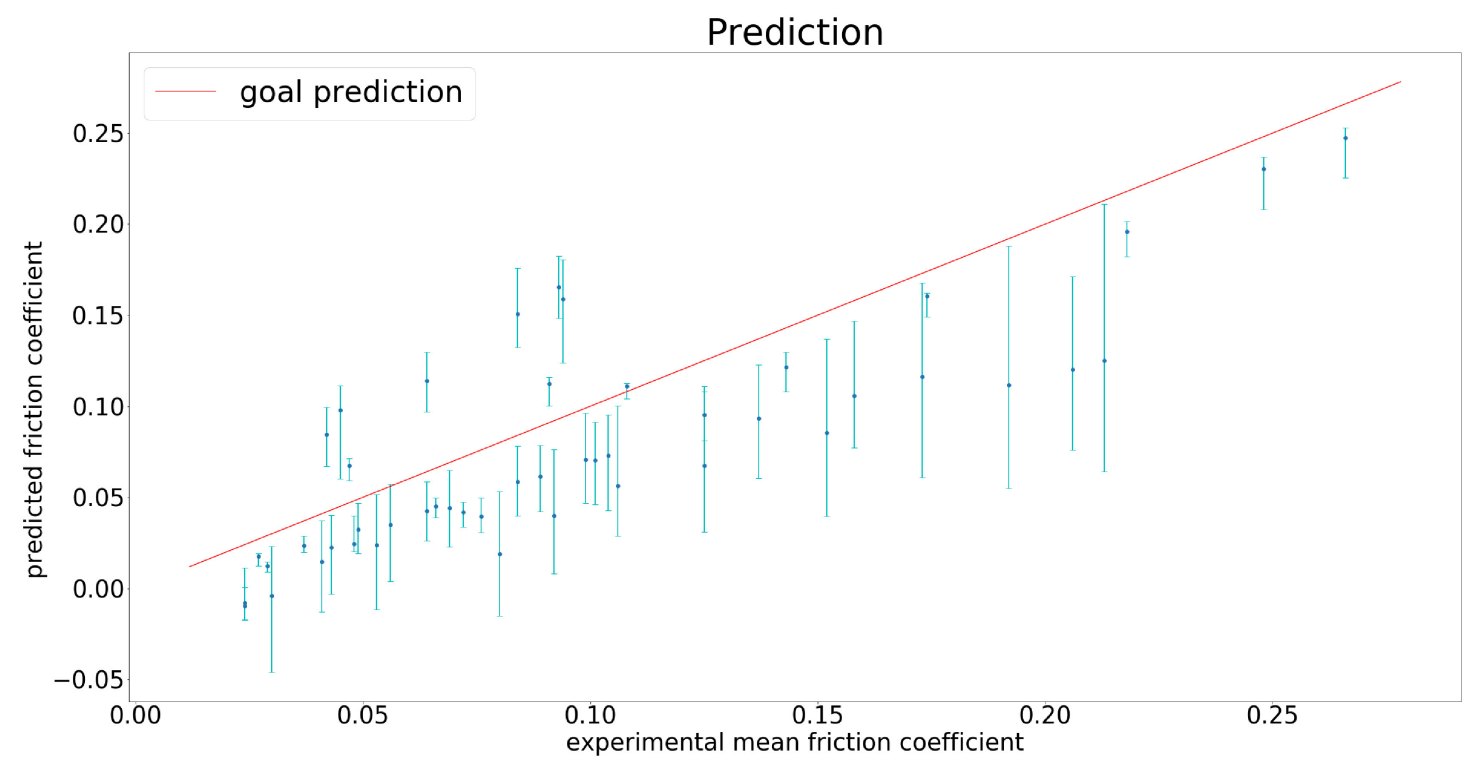

(b)

Figure 12. (a) ROM prediction with statistical noise introduction on depth and (b) on diameter and distance. The blue dots are obtained by evaluating the ROM on the statistically expanded dataset, the solid red line represents the ideal ROM result and the error bars show the friction variations linked to (a) depth or (b) diameter and distance deviations from nominal values.

According to the results shown in Figure 12a,b, when the textured ROM was evaluated on the expanded noisy dataset, friction prediction turned out to be completely affected by the statistical nominal texture values deviations. Once again one can observe that when diameter and distance were made to vary, these variations produced big uncertainties on the predicted friction values and statistically significant differences on mean values, which was corrected when an ideal PDF was used for texture parameters. In order to prove this, friction prediction was repeated using the green distribution in Figure 11a,b, where the texture parameters are centred in their nominal value, with a standard deviation of $\sigma=0.01$. The obtained results are shown in Figure 13a,b. 


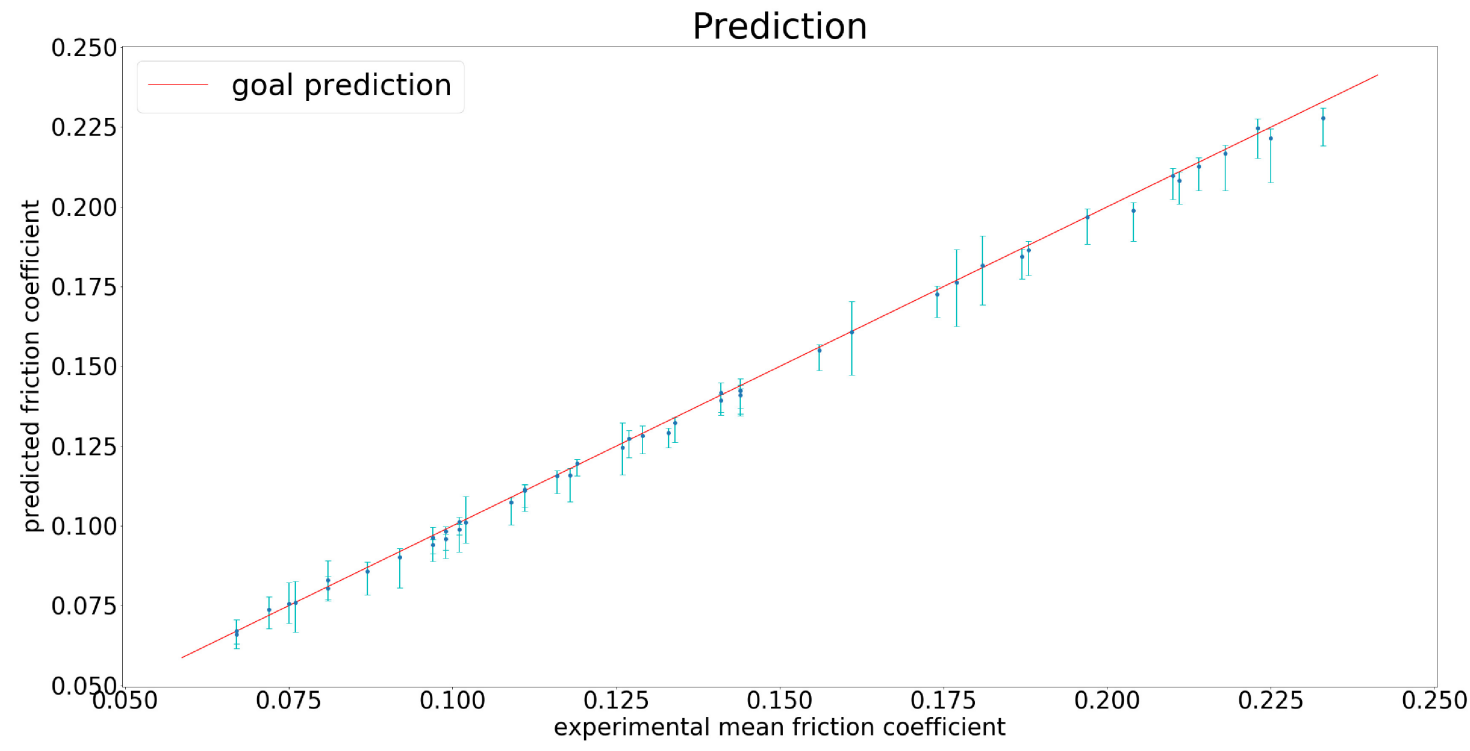

(a)

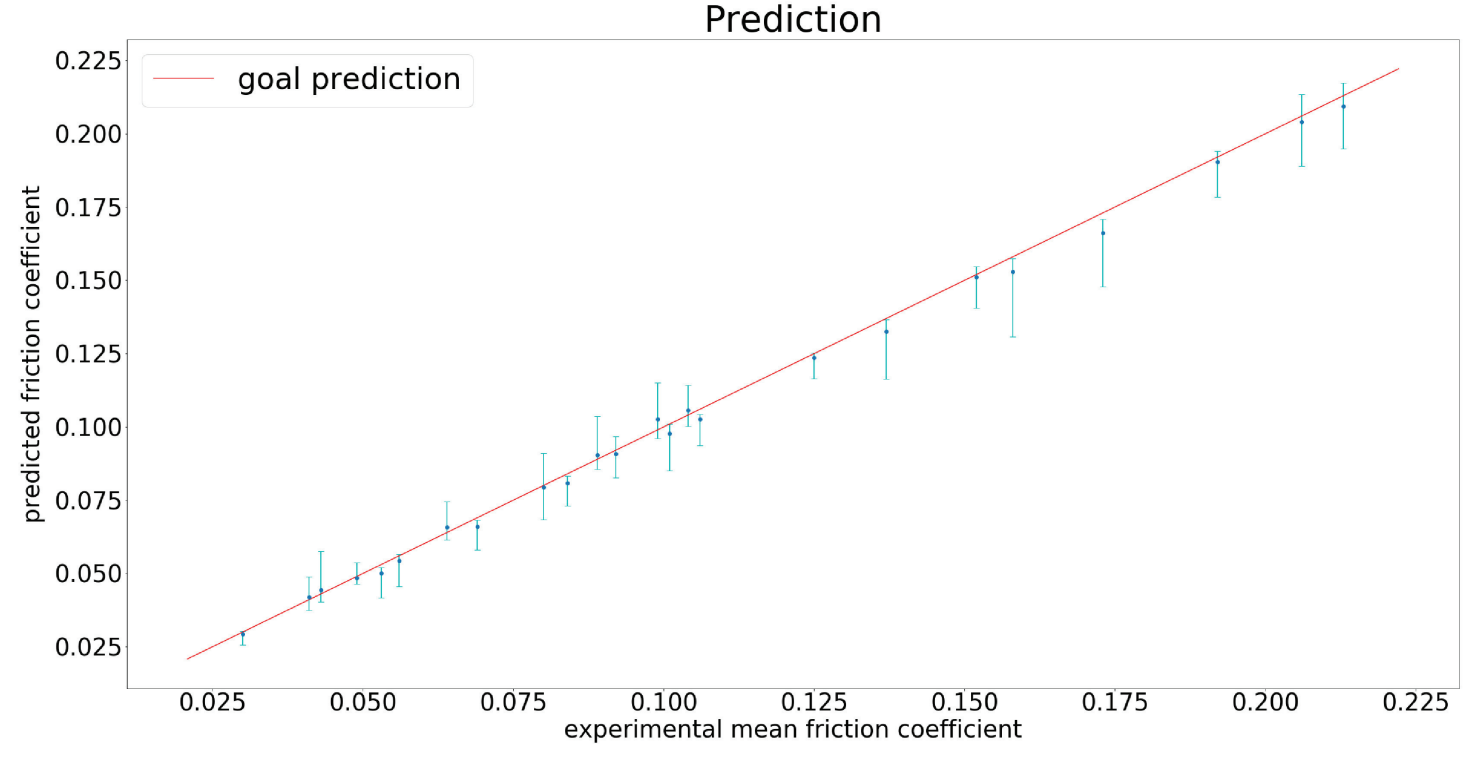

(b)

Figure 13. (a) ROM prediction with statistical noise introduction on depth and (b) on diameter and distance, with centred mean on nominal value and $\sigma=0.01$. The blue dots are obtained by evaluating the ROM on the statistically expanded dataset, the solid red line represents the ideal ROM result and the error bars show the friction variations linked to (a) depth or (b) diameter and distance deviations from nominal values.

\subsection{Experimental Friction Measurement Results and ROM Friction Prediction Outcome}

The objective of the experimental testing procedure is to identify specific surface textures, which exhibit the lowest friction as a function of the relative velocity $v_{r}$ and the contact pressure $p_{c}$. In Figures 14-16 the quasi-stationary friction coefficients $\mu$ of the 8 rubber specimens with different textures are shown as function of the relative velocity $v_{r}$. Data points depicted in Figures 14-16 are based on 5 measurements, whose mean values are shown. Error bars are not provided in the figures due to better visibility, although sigma ranges for the whole pool of data varied between $\sigma_{\min }=0.004$ and $\sigma_{\max }=0.196$ with a mean value of $\sigma_{\text {mean }}=0.005$. 

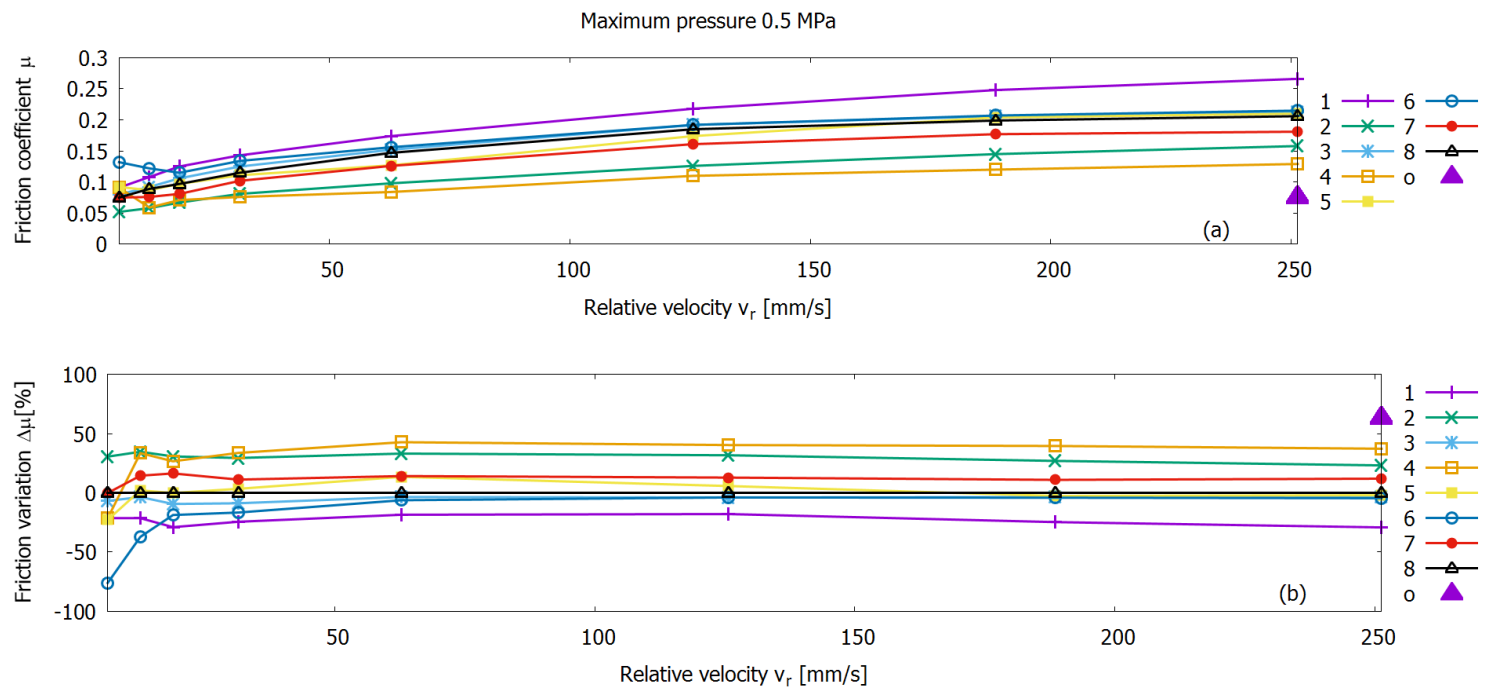

Figure 14. (a) Friction coefficient $\mu$ as function of the relative velocity $v_{r}$ for the eight different rubber specimens and (b) the corresponding friction variation $\Delta \mu$ in relation to the untextured rubber sample 8 , for contact pressures of $p_{c, \text { max }}=0.5 \mathrm{MPa}$.
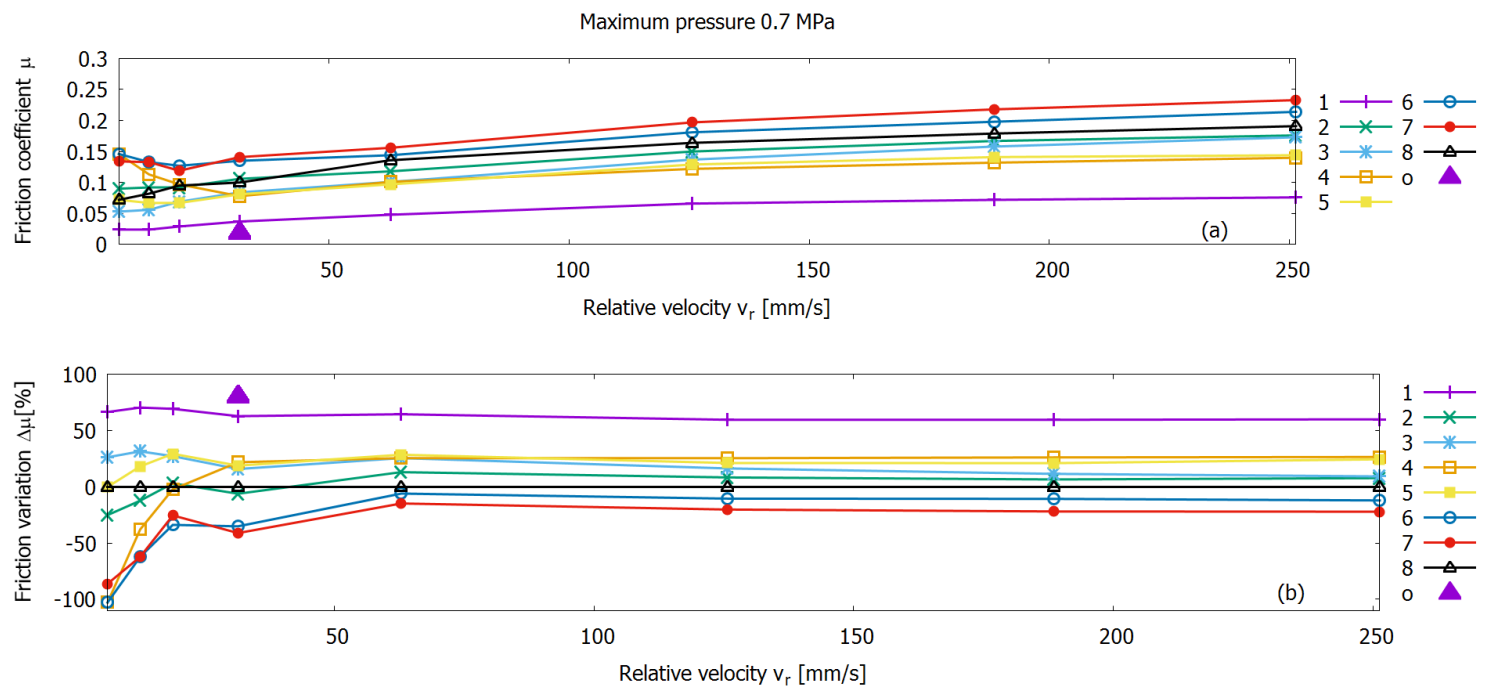

Figure 15. (a) Friction coefficient $\mu$ as function of the relative velocity $v_{r}$ for the eight different rubber specimens and (b) the corresponding friction variation $\Delta \mu$ in relation to the untextured rubber sample 8 , for contact pressures of $p_{c, \max }=0.7 \mathrm{MPa}$.

Each figure visualises the measurement results for one of the three different contact pressures levels $p_{c}$ considered. In addition, the friction variations of the different rubber samples are presented in relation to the untextured reference rubber specimen 8 . Under all operating conditions, friction-reducing, as well as friction-increasing textures, can be identified. Positive values of friction variation indicate a texture-related reduction of friction, while negative friction variation values describe a friction increase compared to the untextured reference sample 8, see also Equation (4). Besides a texture related vertical shift of the friction characteristic, a horizontal shift of the minimum can be observed as well. Hence, surface texturing modifies friction in all regimes, which will be discussed in another publication.

In the following, the textures with the highest and lowest friction are discussed for each contact pressure level $p_{c, \max }$. For $p_{c, \max }=0.5 \mathrm{MPa}$ rubber specimens 2 and 4 exhibit the lowest friction when considering the entire velocity range, while sample 1 reveals the highest friction level. Samples 2 and 4 have the highest area densities of 39\% and 55\%, respectively, while sample 1 has the lowest area density of $9 \%$ within all textures examined. Therefore, a clear trend is evident for $p_{c, \max }=0.5 \mathrm{MPa}$, where the largest area densities 
analysed lead to the lowest friction levels, while the lowest area density leads to the highest friction level. The aspect ratio does not appear to have a major influence in this pressure condition as it fluctuates for samples 1,2 , and 4 between $0.11,0.05$, and 0.10 , so that no clear trend is apparent.
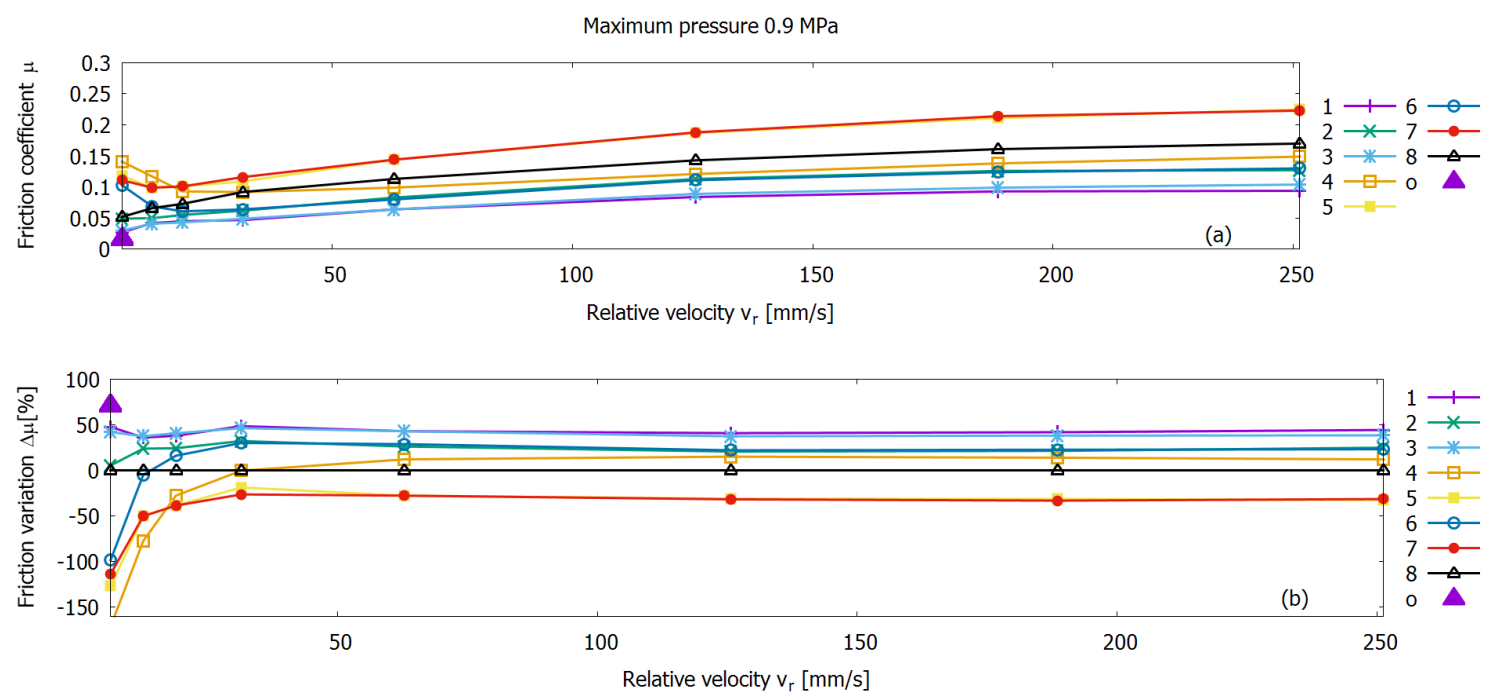

Figure 16. (a) Friction coefficient $\mu$ as function of the relative velocity $v_{r}$ for the eight different rubber specimens and (b) the corresponding friction variation $\Delta \mu$ in relation to the untextured rubber sample 8 , for contact pressures of $p_{c, \text { max }}=0.9 \mathrm{MPa}$.

For $p_{c, \max }=0.7 \mathrm{MPa}$ and $p_{c, \max }=0.9 \mathrm{MPa}$ rubber sample 1 and 3 exhibit the lowest friction level over the entire range of velocities. In contrast, specimen 7 shows the highest friction. Despite this agreement for both pressure levels, no clear trend can be derived in terms of dimple dimensions, area density, or aspect ratio. For example, sample 3 and 7 have similar area density values of $28 \%$ and $25 \%$, respectively, as well as aspect ratios of 0.04 and 0.06 . However, as mentioned above, the measured friction results of both textures differ greatly from each other.

Since there is no clear trend for dimple texture, it is concluded that the texture needs to be individually adapted to the existing operating conditions to minimize friction. For this purpose, ROM is an extremely efficient tool. In order to demonstrate the benefits of the ROM in vivid examples, four exemplary use cases were defined. The first theoretical use case is a friction contact intended to operate at the lowest contact pressure level $p_{c, \max }=0.5 \mathrm{MPa}$ and the highest velocity $v_{r}=251 \mathrm{~mm} / \mathrm{s}$ within the operational conditions experimentally analysed. During the friction measurement procedure, a maximum friction reduction of $37 \%$ was already achieved with sample 4 . However, the theoretical customer requires a further enhanced friction reduction of at least $60 \%$. By using the ROM, a friction reduction of $63 \%$ can be predicted with the texture parameters given in row 1 of Table 5 .

Table 5. Dimple dimensions predicted by the ROM, which further reduce friction based on the use cases.

\begin{tabular}{ccccccccc}
\hline $\begin{array}{c}\text { Use Case } \\
\text { Num. }\end{array}$ & $\begin{array}{c}\text { Relative } \\
\text { Velocity } \\
v_{r}[\mathrm{~mm} / \mathbf{s}]\end{array}$ & $\begin{array}{c}\text { Max Contact } \\
\text { Pressure } \\
p_{c, \max }[\mathrm{MPa}]\end{array}$ & $\begin{array}{c}\text { Dimple } \\
\text { Diameter } \\
{[\mu \mathrm{m}]}\end{array}$ & $\begin{array}{c}\text { Dimple } \\
\text { Distance } \\
{[\mu \mathrm{m}]}\end{array}$ & $\begin{array}{c}\text { Dimple } \\
\text { Depth } \\
{[\mu \mathrm{m}]}\end{array}$ & $\begin{array}{c}\text { Aspect } \\
\text { Ratio }\end{array}$ & $\begin{array}{c}\text { Textured } \\
\text { Area } \\
{[\%]}\end{array}$ & $\begin{array}{c}\text { Predicted } \\
\text { Friction Reduction } \\
{[\%]}\end{array}$ \\
\hline 1 & 251 & 0.5 & 270 & 100 & 10 & 0.04 & 42 & 63 \\
2 & 31 & 0.7 & 300 & 186 & 11 & 0.04 & 30 & 81 \\
3 & 6 & 0.9 & 274 & 111 & 11 & 0.04 & 39 & 72 \\
4 & 100 & 0.6 & 300 & 140 & 11 & 0.04 & 36 & 79 \\
\hline
\end{tabular}

The second use case is an application that operates at a contact pressure $p_{c, \max }=0.7 \mathrm{MPa}$ and a velocity of $v_{r}=31 \mathrm{~mm} / \mathrm{s}$. Friction measurements reveal a maximum friction reduction of $63 \%$ for sample 1 . Again, the friction can be further reduced by a suitable texture, which is 
predicted by the ROM. The ROM indicates a friction reduction of $81 \%$ compared to reference seal 8 for the values specified in row 2 of Table 5 .

The third use case is an imaginary technical requirement for a seal, which operates under the highest contact pressure level of $p_{c, \max }=0.9 \mathrm{MPa}$ and the lowest velocity of $v_{r}=6 \mathrm{~mm} / \mathrm{s}$ within the considered conditions. The experimental friction results show a maximum friction reduction of $48 \%$ for seal sample 1 . With the use of the ROM, a reduced friction of $72 \%$ can be predicted in relation to the untextured case with the values given in row 3 of Table 5.

Based on the ROM results, one additional data point, indicated by a triangle, is added to each of the three plots of Figures 14-16.

In addition to friction predictions, considering the experimentally analysed operating conditions, one of the major advantage of the ROM is the ability to freely interpolate within the parameter space of the operating conditions. This is why the fourth use case shows the $\mathrm{ROM}$ prediction for experimentally untested working conditions, i.e., $p_{c}=0.6 \mathrm{MPa}$ and velocity of $v_{r}=100 \mathrm{~mm} / \mathrm{s}$, where the friction reduction is about $79 \%$, for the texture parameters shown in row 4 of Table 5.

The resulting aspect ratio for all four use case textures is equal to 0.04 , while the textured area ranges from $30 \%$ to $42 \%$. Compared to the experimentally analysed dimple textures given in Table 3, the dimple diameters are rather at the upper edge of the investigated texture dimensions, while the dimple distances and the depths are found in the lower range of the dimple dimensions. However, the dimple values are not similar. Thus, again it is obvious that a surface texture has always to be determined as a function of the operating conditions in order to achieve a maximum friction reduction. For each distinct application and individual technical requirement, textures have to be identified which result in an optimum friction with respect to a defined reference. These ROM results are not experimentally confirmed within the scope of this paper, as it would be necessary to re-produce rubber samples with appropriate textures, which was not part of MouldTex project [39].

\section{Discussion}

As can be concluded from Section 3.5, large texture-induced friction reductions, of up to $70 \%$, could already be found experimentally inside the investigated parameter range of textures, velocities, and contact pressures. However, within the physical available samples, it is not always possible to identify clear trends for dimple dimensions that explain a high friction reduction compared to the untextured reference. Based on this finding, it is inferred that the surface texture dimensions need to be individually adapted to the given operating condition, in order to ensure a low friction level.

One possibility to identify optimal surface textures is the examination of a great number of dimple textures, which is significantly larger than the experimentally analysed set. However, this approach would be time-consuming and expensive due to TDM production requirements. The use of additional methods based on AI, such as ROM, are therefore advantageous. In this context, ROM is an effective method for finding the most suitable textures for specific operating conditions, as shown in Section 3.5, where the ROM is used to predict friction reduction values for both experimentally tested and untested working conditions, see Table 5 rows 1-3 and 4, respectively. Within this paper, ROM models have been used to predict and explore friction behaviour of surface textured rubber specimens by training the model on the experimental friction measurement results. Moreover, the model is able to quantify the measured friction variations that occur when deviations from nominal surface texture values are observed (Figures 9, 10a,b, 11a,b and 12a,b). ROM is extremely useful for simulating and predicting a system behaviour, especially when the physics behind its phenomenology are completely unknown or difficult to solve. Thanks to the ROM algorithms, users can predict the behaviour of their system in real-time, and specifically seal manufacturers can assess the parametric conditions that show the desired optimised results and select them before the rubber seal production. The efficiency of a 
data-driven ROM, as it is for Twinkle [23], is particularly dependent on the collected data, i.e., their associated error and their resulting manifold coverage.

With a thoroughly planned DoE it is possible to achieve an optimal space coverage, which leads to a reliable ROM's prediction result. The promising results, presented in Section 3.3 for the 2-ROM error propagation (Figure 7) strongly support ROM's accuracy on the given dataset. Nevertheless, if larger amounts of data were available, the computed ROM could be even more reliable in those space regions where less information was collected and, hence, confidently retrieve the best texture that allow minimising friction, according to friction variations computed in Equation (4). Moreover, if a ROM model was computed using simulation data, a higher sample space coverage and hence better reliability in the results could be achieved.

In addition, a ROM model allows verifying that the introduction of a statistical noise on nominal surface texture values affects friction, as shown in Figures 11a,b, where the two-tailed t-Student test showed a statistically significant difference between the experimental friction distribution (orange) and the ideal one (green) for dimple diameterdistance variations, see Figures 12a,b, were the difference on friction measurement are linked to manufacturing deviations in the nominal values of dimple textures. These variations were later compared to the effects obtained from an ideal PDF for dimple values experimental variations, i.e., mean value centred on nominal surface texture values and standard deviation $\sigma=0.01$, as shown in Figures 13a,b, opening the way to potential surface texture manufacturing quality and tolerance investigations.

\section{Conclusions}

Within the scope of this paper, a pin-on-disc tribometer was used to measure dynamic friction in the contact of surface textured rubber specimens that were manufactured by texturing during moulding (TDM). The operating conditions, defined by relative velocity $\left(v_{r}\right.$ ranging from 6 to $251 \mathrm{~mm} / \mathrm{s}$ ) and contact pressure level ( $p_{c, \text { max }}$ ranging from 0.5 to $0.9 \mathrm{MPa}$ ), were chosen to correspond to the operating conditions of pneumatic seals. Based on the different experimentally tested texture dimensions, a maximum friction reduction of $70 \%$ was determined compared to the untextured reference, with a dimple diameter of $100 \mu \mathrm{m}$, a dimple depth of $10 \mu \mathrm{m}$ and an area density of $9 \%$.

However, since no global surface texture optimum could be found experimentally that exhibits the lowest friction under all operating conditions, it is concluded that a surface texture needs always to be determined individually, based on the prevailing operating conditions, in order to achieve maximum friction reduction. For this purpose, a novel methodology was applied, combining friction measurements with Reduced Order Modelling (ROM). The objective of the ROM was the computation of optimal surface texture parameters that provide the highest friction reduction within the given parameter space of textures and operating conditions. By feeding the ROM with microscope-based texture measurements, it also takes into account deviations of the real dimple dimensions from the nominal dimple values for the output friction value. In summary, friction measurements are suitable as input parameters for the ROM so that the use of ROM for friction prediction has been proven successful. For demonstration, exemplary use cases were defined, from which it can be concluded that ROM enables the identification of optimal surface texture parameters that were not available for experiments, obtaining friction reductions from $63 \%$ to $81 \%$, which are significantly higher than the experimentally tested surface textures for the same operating conditions. In addition, the value of ROM was further highlighted, as it is also able to freely interpolate between tested operating conditions to determine optimal textures for each operating condition within the range considered, resulting in a predicted friction reduction of $79 \%$.

Moreover, ROM applicability has been extended further, showing that the method can be also used for statistical analysis, to evaluate the impact of manufacturing uncertainties, observed on surface texture nominal values, on friction measurements. Thus, ROM is not only an extremely powerful technique for scientific users to compute the friction reduction 
of surface textured components but also for industrial manufacturers of rubber components, to design rubber surfaces and evaluate the impact of manufacturing deviations on dynamic friction.

Author Contributions: Conceptualization, B.H.-G., M.B. and V.Z.; methodology, B.H.-G. and V.Z.; software, V.Z.; validation, B.H.-G., J.R.V., L.A.G., M.B., M.W. and V.Z.; formal analysis, M.B. and V.Z.; investigation, M.B. and V.Z.; resources, I.V., L.A.G., M.W. and S.I.; data curation, M.B. and V.Z.; writing—original draft preparation, M.B. and V.Z.; writing—review and editing, B.H.-G., J.R.V., L.A.G., M.B., M.W., S.I. and V.Z.; visualization, B.H.-G., J.R.V., L.A.G., M.B., M.W., S.I. and V.Z.; supervision, L.A.G., M.W. and S.I.; project administration, B.H.-G., J.R.V. and L.A.G.; funding acquisition, J.R.V. All authors have read and agreed to the published version of the manuscript.

Funding: This work has been funded by the European Union's research program Horizon2020, under grant agreement No. 768705.

Institutional Review Board Statement: Not applicable.

Informed Consent Statement: Not applicable.

Data Availability Statement: Publicly available datasets were analysed in this study. This data can be found here: http:/ / devex.ita.es/mouldtex/optimizer/Data.zip (accessed on 22 March 2021).

Acknowledgments: We would like to thank ORP Stampi srl and SKM Aeronautics Ltd for providing the rubber specimens and ML Engraving srl for the surface texturing. We would also like to thank Carmen Alfaro, Rafael Rodríguez, and Susana Calvo for useful discussions.

Conflicts of Interest: The authors declare no conflicts of interest.

\section{Abbreviations}

The following abbreviations are used in this manuscript:

AI Artificial Intelligence

CFD Cumulative Density Function

DoE Design of Experiment

DT Digital Twin

FEM Finite Element Method

LST Laser Surface Texturing

ML Machine Learning

PDF Probability Density Function

PGD Proper Generalized Decomposition

POD Proper Orthogonal Decomposition

ROM Reduced Order Modelling

SEHL Soft Elasto-Hydrodynamic Lubrication

SVD Singular Value Decomposition

TDM Texturing During Moulding

TRD Tensor Rank Decomposition

\section{References}

1. Korane, K. Low-Friction Seals Give High Fluid-Power Efficiency. Available online: https:/ /www.machinedesign.com/news/ article/21829567/lowfriction-seals-give-high-fluidpower-efficiency (accessed on 22 March 2021).

2. Jeng, Y.R. Impact of plateaued surfaces on tribological performance. Tribol. Trans. 1996, 39, 354-361. [CrossRef]

3. Wróblewski, P.; Iskra, A. Geometry of shape of profiles of the sliding surface of ring seals in the aspect of friction losses and oil film parameters. Combust. Engines 2016, 55. [CrossRef]

4. Ryk, G.; Kligerman, Y.; Etsion, I. Experimental Investigation of Laser Surface Texturing for Reciprocating Automotive Components. Tribol. Trans. 2002, 45, 444-449. [CrossRef]

5. Tomanik, E.; Profito, F.J; Zachariadis, D.C. Modelling the hydrodynamic support of cylinder bore and piston rings with laser textured surfaces. Tribol. Int. 2013, 59, 90-96. [CrossRef]

6. Etsion, I.; Burstein, L. A Model for Mechanical Seals with Regular Microsurface Structure. Tribol. Trans. 1996, 39, 677-683. [CrossRef]

7. Etsion, I.; Kligerman, Y.; Halperin, G. Analytical and experimental investigation of laser-textured mechanical seal faces. Tribol. Trans. 1999, 42, 511-516. [CrossRef] 
8. Etsion, I. Improving Tribological Performance of Mechanical Components by Laser Surface Texturing. Tribol. Lett. 2004, 17, 733-737. [CrossRef]

9. Pettersson, U.; Jacobson, S. Influence of surface texture on boundary lubricated sliding contacts. Tribol. Int. 2003, 36, 857-864. [CrossRef]

10. Grützmacher, P.G.; Profito, F.J.; Rosenkranz, A. Multi-Scale Surface Texturing in Tribology-Current Knowledge and Future Perspectives. Lubricants 2019, 7, 95. [CrossRef]

11. Shinkarenko, A.; Kligerman, Y.; Etsion, I. The effect of surface texturing in soft elasto-hydrodynamic lubrication. Tribol. Int. 2009, 42, 284-292. [CrossRef]

12. Etsion, I. Modeling of surface texturing in hydrodynamic lubrication. Friction 2013, 1. [CrossRef]

13. Zimmermann, M.; Wangenheim, M. Friction behaviour of surface textured elastomeric seals in pneumatic actuators. In Proceedings of the 23rd International Conference on Fluid Sealing, Manchester, UK, 2-3 March 2016; BHR Group: Cranfield, UK, 2016; p. 97.

14. Dobrica, M.B.; Fillon, M.; Pascovici, M.D.; Cicone, T. Optimizing surface texture for hydrodynamic lubricated contacts using a mass-conserving numerical approach. Proc. Inst. Mech. Eng. Part J J. Eng. Tribol. 2010, 224, 737-750. [CrossRef]

15. Vlădescu, S.; Olver, A.; Pegg, I.; Reddyhoff, T. Combined friction and wear reduction in a reciprocating contact through laser surface texturing. Wear 2016. 358-359, 51-61. [CrossRef]

16. Adjemout, M.; Aurore, A.; Bouyer, J.; Brunetière, N.; Marcos, G.; Czerwiec, T. Influence of the real dimple shape on the performance of a surface textured mechanical seal. Tribol. Int. 2014, 115, 409-416.

17. Tao, F.; Qi, Q.; Wang, L.; Nee, A. Digital Twins and Cyber-Physical Systems toward Smart Manufacturing and Industry 4.0: Correlation and Comparison. Engineering 2019, 5, 653-661. [CrossRef]

18. Tao, F.; Zhang, H.; Liu, A.; Nee, A.Y. Digital twin in industry: State-of-the-art. IEEE Trans. Ind. Inform. 2018, 15, 2405-2415. [CrossRef]

19. Chinesta, F.; Cueto, E.; Abisset-Chavanne, E.; Duval, J.; Khaldi, F. Virtual, Digital and Hybrid Twins: A New Paradigm in Data-Based Engineering and Engineered Data. Arch. Comput. Methods Eng. 2018. [CrossRef]

20. Le Clainche, S.; Vega, J.M. A Review on Reduced Order Modeling using DMD-Based Methods. In Proceedings of the IUTAM Symposium on Model Order Reduction of Coupled Systems, Stuttgart, Germany, 22-25 May 2018; Springer: Berlin/Heidelberg, Germany, 2020; pp. 55-66.

21. Mignolet, M.P.; Przekop, A.; Rizzi, S.A.; Spottswood, S.M. A review of indirect/non-intrusive reduced order modeling of nonlinear geometric structures. J. Sound Vib. 2013, 332, 2437-2460. [CrossRef]

22. Wuest, T.; Weimer, D.; Irgens, C.; Thoben, K.D. Machine learning in manufacturing: Advantages, challenges, and applications. Prod. Manuf. Res. 2016, 4, 23-45. [CrossRef]

23. Zambrano, V.; Rodríguez-Barrachina, R.; Calvo, S.; Izquierdo, S. TWINKLE: A digital-twin-building kernel for real-time computer-aided engineering. SoftX 2020, 11, 100419. [CrossRef]

24. Dataset Generated and Used Within This Publication. Available online: http://devex.ita.es/mouldtex/optimizer/Data.zip (accessed on 22 March 2021).

25. Lee, C.Y.; Lin, C.S.; Jian, R.Q.; Wen, C.Y. Simulation and experimentation on the contact width and pressure distribution of lip seals. Tribol. Int. 2006, 39, 915-920. [CrossRef]

26. Parker Pneumatic Seals Catalogue. Available online: https://www.parker.com/Literature/Praedifa/Catalogs/Catalog_ PneuSeals_PTD3351-EN.pdf (accessed on 22 March 2021).

27. OKS Adherent Silicone Grease Lubricant Specification. Available online: https://www.oks-germany.com/en/products/oks-11 55-adherent-silicone-grease/ (accessed on 22 March 2021).

28. TWINKLE: A Digital-Twin-Building Kernel for Real-Time Computer-Aided Engineering. Available online: https://github.com/ caeliaITAINNOVA/Twinkle (accessed on 22 March 2021).

29. Pedregosa, F.; Varoquaux, G.; Gramfort, A.; Michel, V.; Thirion, B.; Grisel, O.; Blondel, M.; Prettenhofer, P.; Weiss, R.; Dubourg, V.; et al. Scikit-learn: Machine Learning in Python. J. Mach. Learn. Res. 2011, 12, 2825-2830.

30. Langford, J. The cross validation problem. In Proceedings of the International Conference on Computational Learning Theory, Bertinoro, Italy, 27-30 June 2005; Springer: Berlin/Heidelberg, Germany, 2005; pp. 687-688.

31. Bochkanov, S. ALGLIB. Available online: www.alglib.net (accessed on 22 March 2021).

32. Ayodele, T.O. Types of machine learning algorithms. New Adv. Mach. Learn. 2010, 3, 19-48.

33. Berry, M.W.; Mohamed, A.; Yap, B.W. Supervised and Unsupervised Learning for Data Science; Springer: Berlin/Heidelberg, Germany, 2019.

34. Harris, C.R.; Millman, K.J.; van der Walt, S.J.; Gommers, R.; Virtanen, P.; Cournapeau, D.; Wieser, E.; Taylor, J.; Berg, S.; Smith, N.J.; et al. Array programming with NumPy. Nature 2020, 585, 357-362. [CrossRef]

35. Virtanen, P.; Gommers, R.; Oliphant, T.E.; Haberland, M.; Reddy, T.; Cournapeau, D.; Burovski, E.; Peterson, P.; Weckesser, W.; Bright, J.; et al. SciPy 1.0: Fundamental Algorithms for Scientific Computing in Python. Nat. Methods 2020, 17, $261-272$. [CrossRef]

36. Shinkarenko, A.; Kligerman, Y.; Etsion, I. The Validity of Linear Elasticity in Analyzing Surface Texturing Effect for Elastohydrodynamic Lubrication. Trans. ASME J. Tribol. 2009, 131. [CrossRef] 
37. Wang, X.; Kato, K.; Adachi, K.; Aizawa, K. The effect of laser texturing of SiC surface on the critical load for the transition of water lubrication mode from hydrodynamic to mixed. Tribol. Int. 2001, 34, 703-711. [CrossRef]

38. Schneider, J.; Braun, D.; Greiner, C. Laser Textured Surfaces for Mixed Lubrication: Influence of Aspect Ratio, Textured Area and Dimple Arrangement. Lubricants 2017, 5, 32. [CrossRef]

39. Mouldtex Project. Available online: http:/ / www.mouldtex-project.eu/ (accessed on 22 March 2021). 